QUARTERLY OF APPLIED MATHEMATICS

VOLUME LXV, NUMBER 2

JUNE 2007, PAGES 205-257

$\mathrm{S} 0033-569 \mathrm{X}(07) 01028-8$

Article electronically published on May 1, 2007

\title{
ON THE MATHEMATICS OF GROWTH
}

\author{
BY \\ ULF GRENANDER \\ Division of Applied Mathematics, Brown University, Providence, Rhode Island 02912 \\ Dedicated to d'Arcy Thompson in memory of a great pioneer, 1860-1948
}

1. A mathematical challenge to biology. It is well known that research in biology/medicine has come to rely extensively on computers, software and algorithms. But how about the influence of mathematics? If we had asked the same question for physics the answer would be a resounding YES: physics has clearly flourished under the impact of mathematical thinking. Well, it was not always so. Indeed, there was a time when it was admitted that mechanics was well suited for mathematical analysis, using for example Newton's calculus, while other subfields, such as magnetism and heat, were considered essentially too complicated for mathematical analysis. The experimental physicists resisted the theorists, which can be seen by reading the discussions of the Nobel prize committee even as late as in the 1930s.

But we know what happened! What followed was an overwhelming triumph for mathematics in understanding physics. Is the same development going to take place in biology/medicine? Of course medical researchers are used to employing statistics, analysis of variance and other traditional tests, but we are thinking of less pedestrian applications supplying a basis for basic biological understanding. Actually, mathematics has been applied to biology for many years, but it must be admitted that real, or wet biologists, have often been skeptical, sometimes with good reason. One of the pioneers in mathematical biology, Nicholas Rashevsky, had a grand plan $\tilde{n}$ to create a field of mathematical biophysics, which was presented in his ambitious work "Mathematical Biophysics" published in 1938. What a wonderful idea! This author was certainly impressed by it when it appeared. But professional biologists were less impressed and the work seems to have had little impact. What was wrong? Now, much later, it is clear that the early attempts of mathematical formalization contained too little subject matter substance, too little biology, and this was the reason why it was met by some healthy skepticism. Today, 70 years later, the mental climate is quite different; in some fields of medicine we can

Received February 21, 2006.

2000 Mathematics Subject Classification. Primary 92B05.

This work has been supported by NSF DMS-00774276.

(C)2007 Brown University 
see mathematical methods applied with some success, especially among young medical researchers.

There are many success stories. One example is population genetics; see Lewontin (1974). This mathematics actually works and has a firm foundation in Mendelian theory. It is not just curve fitting but penetrates the essence of the problem. Another example is the Hodgkin-Huxley model for exchange of sodium and potassium ions that causes a brief reversal in a nerve cell's electrical polarization resulting in the transmission of an impulse along a nerve fibre; see Hodgkin, Huxley (1952). Again, this success is anchored in well-established neurophysiological doctrine. These examples, two of many, illustrate the power of mathematical modelling if it is based on sound biological principles, and this shall be our guide in what follows.

2. Especially image processing. For concreteness we shall narrow the discourse to medical image processing, more precisely to computational anatomy. One school in the field of computational anatomy has relied systematically on random mappings to represent deformations of templates in order to represent biological variation in mathematical terms. This has been done especially for static situations when the observation is taken at a single time point; see Miller (2004), Miller, Joshi and Christensen (1999), Grenander, Miller (1998), Thompson and Toga (2002), Beg, Miller, Trouve, Younes (2002).

To fix ideas we shall formalize the problem as follows, saying that the organism $\Omega$ to be observed is contained in a region $X$ in the plane or 3-space and is observed by some camera device, perhaps employing visible light or MR, magnetic resonance. This results in an image $I^{\mathcal{D}}$, scalar or vector valued. The goal is to use $I^{\mathcal{D}}$ in order to make inferential statements about $I$.

Let us think of $I^{\mathcal{D}}$ as the result of a cascade of transformations of $I$; see Matejic (1997). For example, the group cascade could be

1) the groups $\mathrm{SE}(2)$ or $\mathrm{SE}(3)$, possibly augmented with scale change; registration

2 ) the multiplicative group on $\mathbf{R}+$; photometric effect, perhaps gamma correction

3) the groups $\operatorname{DIFFEO(2)~or~} \operatorname{DIFFEO(3);~normal~biological~variation~}$

4) the semi-groups CREATION, ANNIHILATION representing pathologies

5) an additive stochastic process; camera noise.

In a Bayesian mode we introduce probability measures on the transformations to represent the various causes of variability. This can be said to be an anatomical textbook in digital form. But this can be done in many ways, on different levels of ambition, so let us look at just a few. A diagnostical aid can then be obtained by inference from the posterior probability measure, at least in principle.

2.1. Small deformations. Replacing probabilities with energies, $E=-\log p$, we shall combine energies from 1), 2), 3), 4), 5); for example,

$$
E_{3} \propto \int_{X}\|\operatorname{grad} \phi\|^{2} d x
$$

where

$$
E_{5} \propto \int_{X}\left[\phi^{-1} \circ I-I^{\mathcal{D}}\right]^{2} d x
$$


for the additive noise superimposed on the developed organism $\phi^{-1} \circ I$. The form of the other energies will depend upon the specifics of the image acquisition.

Add up all the relevant energies and apply MAP (Maximum Aposterior Probability) or some other optimality to estimate the mapping $\phi^{*}$. Other inferences, for example determination of anatomical components in $I^{\mathcal{D}}$, can be derived from $\phi^{*}$. Such procedures work fairly well if the biological deformation is small. In the opposite case, however, the mapping may be nonbijective and hence biologically inadmissible.

2.2. Large deformations. In the latter case some modification of the above is neccessary and an appealing alternative is offered by letting the deformations be generated by flows. This could be done for example as follows:

$$
\frac{\partial[x(t)]}{\partial t}=v[x(t)] ; \quad t_{1}<t<t_{2}
$$

for the flow of a point $x \in X$ during the time duration $\left(t_{1}, t_{2}\right)$, and with a velocity $v$ controlled by the instantaneous energy

$$
E_{3} \propto \int_{X}[(L v)(x)]^{2} d x
$$

that can be used to derive the Euler equations for the optimality problem. This approach has proven successful in medical imaging.

3. Dynamic case. When we turn to the dynamic case of growth we believe that one should employ a more detailed representation built on fundamental biological facts expressing the atomistic nature of growth in terms of units, the cells, that are subject to decisions controlling events such as mitosis, cell death, cell movement and other primitive changes. These events are composed with each other, resulting in iterated mappings that are more specific than diffeomorphic maps generally, but will still be contained in the full diffeomorphic group. In this way we hope that the model will be sufficiently realistic. But we shall also insist on using a biologically motivated coordinate system rather than the standard Cartesian one: organisms in embryology do not know absolute space.

3.1. Requirements for growth models. A mathematical model of biological growth must be based on principles consistent with what is known about growth phenomena, relying on both anatomical and genetic basics. It is tempting to borrow familiar mathematical tools from differential equations and probability theory to build the model, perhaps appealing to ideas from fluid mechanics, but if this is not accompanied by a critical biological scrutiny, we run the risk of just playing a mathematical game.

Whatever are the laws underlying the development of an organism it seems clear that they should be expressed with some invariance: the organism does not know absolute space (but may of course know its environment) and its decisions are made in terms of an intrinsic coordinate system. We therefore formulate the following requirement. If the organism $\Omega$ at time $t$ occupies a region $\Omega(t) \subset \mathbf{R}^{2}$, then its intrinsic coordinate system should be defined inside $\Omega(t)$.

A BIOLOGICAL COORDINATE SYSTEM. The development equations shall be formulated in terms of a relative coordinate system independent of absolute space, but related to the anatomy of the organism. 
The genetic information that governs the development is given at the beginning $t=0$ of the growth cycle although during the development genes will be turned on and off by a program written in the language of the biological coordinate system. As time goes on the genes follow the changes in the coordinate system as it moves and deforms in absolute space. Hence

GENETICS EXPRESSED VIA THE BIOLOGICAL COORDINATE SYSTEM. The genes are functions of the biological coordinates and of (relative) time.

As the organism expands or contracts, changing its shape and undergoing internal changes, it cannot overlap itself, i.e., volumes exclude each other. A model that allows overlap is not acceptable. Thus

EXCLUSION OF VOLUMES. The mappings that transform the organism must geometrically be diffeomorphic.

Organisms are built from cells - an atomistic statement. Any realistically detailed model must therefore be constructed from units, so that we have

DISCRETE STRUCTURE. The model should be discrete with a finite number of units.

On the other hand, the units are small macroscopically and there are many of them. This invites a mathematical analysis with the flavor of the law of large numbers and asymptotics.

LARGE NUMBER OF UNITS. $\Omega$ shall consist of many biological units.

The development is influenced by the environment and by changes in the genetic setup. This leads us to probabilistic models.

NON-DETERMINISTIC LAWS. The dynamics of development will have random elements.

4. Darcyan coordinates. The most obvious coordinate system may be a cartesian system oriented with respect to significant features, land marks, in the anatomy. For the brain this can lead to the celebrated Talairach system, oriented with respect to the anterior commissures and with axes in the left-right, posterior-anterior, and ventraldorsal directions; this is a system in current use among radiologists. Our study, however, will be expressed in a coordinate system that mirrors the anatomical shape more directly and is a carrier of genetic codes.

To chart the spatial gene distribution we shall introduce a Darcyan coordinate system, named after d'Arcy Thompson, with coordinates $\xi=\left(\xi_{1}, \xi_{2}\right) \subset \Xi$, such that the points in $\Omega(0)$ are given as a function $x\left(\xi_{1}, \xi_{2}\right)=\left[x_{1}\left(\xi_{1}, \xi_{2}\right), x_{2}\left(\xi_{1}, \xi_{2}\right)\right]$. As the organism develops, the Darcyan coordinate system too will develop. The Darcyan is usually curvilinear in $\mathbf{R}^{2}$ and can look like the diagram in Figure 1. The green lines denote the level sets of the radial variable $\xi_{1}$, while the dotted lines denote the level sets of the angular variable $\xi_{2}$.

Note that we are working with both an absolute Cartesian coordinate system $\left(x_{1}, x_{2}\right)$ and a time-dependent relative coordinate system $\left(\xi_{1}, \xi_{2}\right)$. All biological decisions during development will be expressed in Darcyan terms and relative space. The time parameter $t$ measures time from the instant when $\Omega(0)$ is observed, possibly birth.

Therefore, in the GRID representation a central role is played by the Darcyan: a coordinate system that develops in time together with the organism it represents. It 
is essential that the coordinate system mirror the gross anatomy of the organism, emphasizing its boundary. As our standard choice in our illustrations we have used a disk described by polar coordinates $\xi=\left(\xi_{1}, \xi_{2}\right)$. More precisely, we have studied mappings

$$
\phi:\left\{0<\xi_{1}<\infty\right\} \times\left\{0<\xi_{2}<2 \pi\right\} \rightarrow \mathbf{R}^{2} \backslash\{0\} .
$$

Note that we have excluded the origin that would otherwise be a singular point.

But this was only intended as an illustration. In general we shall introduce Darcyan geometries given through Riemannian metrics of the form

$$
(d s)^{2}=\sum_{i, j} g_{i j} d \xi_{i} d \xi_{j}
$$

with the covariant metric tensor $g=\left\{g_{i j}\right\}$. Then it is well known that the Laplacian can be written as

$$
\Delta \phi=\frac{1}{\sqrt{g}} \sum_{i, j} \frac{\partial}{\partial \xi_{i}}\left(\sqrt{g} g^{i j} \frac{\partial \phi}{\partial \xi_{j}}\right)
$$

using the contravariant tensor $\left\{g^{i j}\right\}$.

As the organism grows the metric tensor changes too. In the isotropic GRID case the thermodynamic equation (see sections 5.2 and 5.3) can be written as

$$
\frac{\partial x(\xi, t)}{\partial t}=\int_{\eta \in \Xi}[x(\xi, t)-x(\eta, t)] \psi\left(\frac{1}{2}\|x(\xi, t)-x(\eta, t)\|^{2} A(d \eta)\right.
$$

with some growth decay function $\psi$. Then we get

$$
\begin{aligned}
\frac{\partial g_{11}}{\partial t}= & \int_{\eta \in \Xi}\left\{g_{11} \psi\left(\frac{1}{2}\|x(\xi, t)-x(\eta, t)\|^{2}\right)\right. \\
& +\left[x_{1}(\xi, t)-x_{1}(\eta, t)\right] \psi^{\prime}\left(\frac { 1 } { 2 } \| x ( \xi , t ) - x ( \eta , t ) \| ^ { 2 } \left(\left[x_{1}(\xi, t)-x_{1}(\eta, t)\right] g_{11}\right.\right. \\
& \left.\left.+\left[x_{2}(\xi, t)-x_{2}(\eta, t)\right] g_{21}\right)\right\} A(d \eta)
\end{aligned}
$$

and similar expressions for the time derivatives of the other members of the metric tensor. This becomes, written in tensor form,

$$
\frac{\partial g}{\partial t}=\int_{\eta \in \Xi}\left[\psi\left(\frac{1}{2}\|x(\xi, t)-x(\eta, t)\|^{2}\right) i d(2)+\psi^{\prime}\left(\frac{1}{2}\|x(\xi, t)-x(\eta, t)\|^{2}\right) V\right] g A(d \eta)
$$

with $V$ a $3 \times 3$ matrix

$$
V=\left(\begin{array}{cc}
v_{1}^{2} & v_{1} v_{2} \\
v_{2} v_{1} & v_{2}^{2}
\end{array}\right)
$$

and

$$
v_{1}=x_{1}(\xi, t)-x_{1}(\eta, t) ; \quad v_{2}=x_{2}(\xi, t)-x_{2}(\eta, t) .
$$

The expression $i d(2)$ stands for the identity in 2 -space. This equation describes the development of the biological geometry as $\Omega(t)$ is growing.

We shall be especially interested in the orthogonal case when the tensors are of diagonal type and the above simplifies to

$$
(d s)^{2}=\sum_{i}\left(h_{i} d \xi_{i}\right)^{2} ; \quad h_{i}=\sqrt{g_{i i}}=\sqrt{\left(\frac{\partial x_{1}}{\partial \xi_{1}}\right)^{2}+\left(\frac{\partial x_{2}}{\partial \xi_{2}}\right)^{2}}
$$


and

$$
\Delta \phi=\frac{1}{\prod_{i} h_{i}} \sum_{i} \frac{\partial}{\partial \xi_{i}}\left(\frac{\prod_{j} h_{j}}{h_{i}} \frac{\partial \phi}{\partial \xi_{i}}\right) .
$$

For example, the Darcyan mentioned above with $\xi=\left(\xi_{1}, \xi_{2}\right)=(r, \theta)$, leads to the classical expressions

$$
(d s)^{2}=(d r)^{2}+r^{2}(d \phi)^{2}
$$

and

$$
\Delta \phi=\frac{1}{r^{2}} \frac{\partial}{\partial r}\left(r^{2} \frac{\partial \phi}{\partial r}\right)+\frac{1}{r^{2} \sin \theta} \frac{\partial}{\partial \theta}\left(\sin \theta \frac{\partial \phi}{\partial \theta}\right) .
$$

But how do we construct an initial (later changing over time) Darcyan for a set $X$ of general shape, say compact, connected and simply connected, with a smooth boundary? In the plane we could simply choose a conformal map between the set $X$ and the unit disk with coordinates corresponding to the concentric circles and equally spaced radii. In 3-space we do not have access to such mappings and will have to look elsewhere for a construction of a Darcyan.

Here is one suggestion. Select an interior point $P$, a pole, in $X \subset \mathbf{R}^{3}$ and solve the Poisson equation with Dirichlet boundary conditions

$$
\Delta u=\delta_{P} ; u=0 \quad \text { on } \quad \delta X,
$$

where we have placed a unit charge $\delta_{P}$ at the pole $P$. (We actually think of solving the Poisson equation in the region excluding a small disk centered at $P$ and a positive boundary value on its circumference; then let the radius of the disk tend to zero.) Introduce the level surfaces $\{u=$ constant $\}$ and parametrize them. Actually, it will be convenient to scale the $u$-values nonlinearly so that $u=0$ at the boundary and approaches $u=1$ close to the pole. Also consider the trajectories starting at the boundary and proceeding in the direction of the gradient $\operatorname{grad} u$ toward $P$. Then for any interior point use the level value of $u$ together with the $2 \mathrm{D}$ value of the point on the boundary for the associated trajectory to construct Darcyan coordinates. Unfortunately we cannot guarantee that for 3D each interior point is passed through by exactly one trajectory; according to Khavinson (2005) this question has not yet been resolved. For the time being we therefore have to consider this suggestion only as a tentative solution.

Returning to the 2D case let us think of a general anatomical map where the organism contains sub-organisms containing sub-sub-organisms and so on. All these sets constitute a tree $\mathbf{T}$ that we arrange on levels according to the way inclusions are ordered, $l=$ $0,1,2, \ldots$ We get $2 \mathrm{D}$ sets, organism $X^{0}$, sub-organisms $X_{1}^{1}, X_{2}^{1}, \ldots \subset X^{0}$, sub-sub-sets $X_{1}^{2}, X_{2}^{2}, \ldots$ and so on. The organism in Figure 2a, diagrammed in Figure 2, leads to the anatomical chart in Figure 3 so that we are dealing with the tree structure.

Then we solve Poisson's equation in a region $X_{i}$ with an outside boundary $B_{\text {outside }}=$ $\partial_{\text {outside }} X_{i}$ and an inside $B_{\text {inside }}=\partial_{\text {inside }} X_{i}$ with boundary values $V_{i}$ on $B_{\text {outside }}$ and $V_{i}+1$ on $B_{\text {inside }}$. For an organism with two sub-organisms the level curves can look like Figure 4 in the region just inside the outer boundary of the organism; compare values with the color bar. 
The other coordinate, the angular one, will be orthogonal to the level curves. A moment's reflection shows that the coordinate set of any sub-organism will conveniently be in the form of the product $\Xi_{k}^{1} \times \Xi_{l}^{2}$. This fact should help in building computational anatomical atlases.

The $\xi_{2}$ axes will be curves from the boundary toward the singular point. More precisely, the Darcyan coordinates can be constructed as follows. Let the boundary $\partial X$ be parametrized as $x(s), 0<s<L$ in terms of arc length. Define the angular coodinate as $\xi_{2}=s$. Then solve for fixed $\xi_{2}$ the ODE

$$
\frac{d x\left(t, \xi_{2}\right)}{d t}=\frac{\operatorname{grad} u}{\|\operatorname{grad} u\|}, 0<t<1,
$$

and put $\xi_{1}=t$ along the solution curve. Note that, at least in 2D, the $u$-field has no singular points outside the pole so that the gradient does not vanish and the ODE is meaningful.

As an example we show in Figure 5 a slice of a brain in (a) together with the Darcyan in (b) induced by a version of the above procedure.

Given a Darcyan in $\Xi$ we shall introduce an orthonormal system $\mathcal{F}$ of functions $f_{1}(\xi), f_{2}(\xi), f_{3}(\xi), \ldots$ such that

$$
\int_{\Xi} f_{i}(\xi) f_{j}(\xi) d \xi=\delta_{i j}
$$

A natural way of creating $\mathcal{F}$ is to start with functions in the form of the product $g_{n}\left(\xi_{1}\right)$ $h_{m}\left(\xi_{2}\right), n, m=1,2,3 \ldots$ and then apply the Gram-Schmidt orthogonalization to them.

The idea of biological coordinate systems was clearly enunciated in d'Arcy Thompson's admirable book "Growth and Form" (1917). His "method of coordinates" is certainly the starting point for studies in the spirit of this paper. It is true that Thompson did not have access to the mathematics needed to give a solid quantitative foundation to his ideas, but with remarkable intuitive insight he formulated the basic principles that underlie mathematical understanding of biological growth. In this connection we should mention the pioneering work of Bookstein, see Bookstein $(1978,1997)$, whose statistical analysis of biological shape has attracted much attention.

It may be useful to apply a nonlinear transformation to the solution of Poisson's equation in order to get a coordinate system that is more evenly distributed over $X$ than some shown below.

Let us finally quote d'Arcy Thompson:

"in the study of material things number, order, and position are the threefold clue to exact knowledge: and that these three, in the mathematician's hands, furnish the first outlines for a sketch of the Universe."

\section{GRID synthesis.}

5.1. Iterated diffeomorphisms. Let $\phi$ be a diffeomorphism from $\mathbf{R}^{2}$ to itself, and let the point represented by $x(\xi)$ in the Darcyan $\xi \in \Xi$ be moved to $\phi(x(\xi))$ under the mapping $\phi$. We restrict the space of allowable deformations by using a seed-based definition that we shall sometimes denote by $\phi^{\alpha}$. Shown in Figure [ is a seed $x\left(\xi_{\text {seed }}\right)$, indicated with 
a small red star, and the point being considered for updating is denoted by $x(\xi)$. The resulting Darcyan is shown in the right panel for a standard coordinate system.

Hence we are dealing with an organism $\Omega$, living in the background space $X=\mathbf{R}^{3}$ and developing as a function of time, $\Omega(t)$. It is made up of disjoint components $\Omega=\bigcup g^{\alpha}$ such that each generator (anatomical component) $g^{\alpha}$ is fairly homogeneous w.r.t. growth properties as controlled by the genetics; examples will be given later.

The organism grows as expressed by a composition hemi-group $\Phi=\{\phi(s, t) ;-\infty<$ $s<t<\infty\}$ of diffeomorphisms from DIFFEO(X);X $=\mathbf{R}^{3}$ so that

(i) $\phi\left(t_{1}, t_{2}\right) \circ \phi\left(t_{2}, t_{3}\right)=\phi\left(t_{1}, t_{3}\right), t_{1}<t_{2}<t_{3}$;

(2) $\phi(s, t)$ is continuous in $s, t$ (topology to be decided later);

(3) $\phi(t, t)=i d(X)$.

The behavior of the mapping $x \rightarrow \phi(x)$ from a point $x \notin \Omega(s)$ at time $s$ is irrelevant. Growth will be represented as

$$
\Omega(t)=\phi^{-1}\left(t_{0}, t\right) \Omega\left(t_{0}\right), t_{0} \text { fixed. }
$$

It will be assumed that the $\alpha$-partition is invariant w.r.t. the $\phi$ 's so that the genetic properties remain constant along trajectories induced by $\Phi$.

Consider the Galilean space-time group

$$
S=\{-\infty<t<\infty\} \times S E(3)
$$

with elements generically denoted by $s$. The set of all growths $(t, \phi(\cdot, \cdot))$ invariant with respect to $S$ is called a growth pattern; see Grenander (1993) for pattern-theoretic terminology. But we can also deal with other growth patterns, for example by replacing $S E(3)$ in (1) by DIFFEO(X).

The space-time coordinate system $\{-\infty<t<\infty\} \times X$ is absolute in contrast to the biological coordinate system that we shall use:

$$
\{0<\tau<\infty\} \times \Xi
$$

meaning that we measure time for $\Omega$ from its birth $\tau=0$ and location on $\Omega(\tau)$ by the value $\xi$ of $x$ at time 0 , where we use Lagrangian coordinates and consider the trajectories in absolute space to be denoted by $x(\tau)=\phi\left(t_{0}, \tau\right) \xi$, where $\xi$ enumerates the trajectories with the initial values $x(\xi, 0)=\xi \in \Xi$. Since we are dealing with diffeomorphisms the function $x=x(\xi, \tau)$ has a well-defined inverse $\xi=\xi(x, \tau)$.

The elementary events that govern a growth process will be chosen as the discrete decisions of cells as controlled by those of their genes that are switched on at the moment. This could be e.g. mitosis, corresponding to proper growth, or cell death meaning decay. Let the active genetic setup of a cell located at Darcyan coordinate $\xi$ at time $t$ be denoted by a switching function

$$
\alpha=\alpha(\xi, t) .
$$

Hence $\alpha\left(\xi_{\text {seed }}, t\right)$ denotes the nature of the deformation at location $\xi_{\text {seed }}$ at time $t$. Note that its spatial dependence is in terms of Darcyan coordinates, not in an absolute and fixed coordinate system. An elementary event at age $t$ due to a cell decision at $\xi_{\text {seed }}$ causes a change in the $x$-field through a diffeomorphism:

$$
\phi^{\alpha\left(\xi_{\text {seed }}, t\right)}: x(\xi) \mapsto \phi^{\alpha\left(\xi_{\text {seed }}, t\right)}(x(\xi)) .
$$


Hence a sequence of events at ages $t_{1}, t_{2}, \ldots, t_{n}$ at the corresponding seed locations $\xi_{\text {seed }}^{1}, \xi_{\text {seed }}^{2}, \ldots, \xi_{\text {seed }}^{n}$ lead to an iterated diffeomorphism

$$
\Phi=\phi^{\alpha\left(\xi_{\text {seed }}^{n}, t_{n}\right)} \circ \ldots \circ \phi^{\alpha\left(\xi_{\text {seed }}^{2}, t_{2}\right)} \circ \phi^{\alpha\left(\xi_{\text {seed }}^{1}, t_{1}\right)}
$$

to be applied to the original value of the $x$-field. In view of the random placements of seeds, and the random nature of the diffeomorphisms, characterized by the $\alpha$ 's, the elementary cell divisions will be random in nature; see Section 5.2. This is the Growth Random Iterated Diffeomorphism model - the GRID model - in its general form. We shall specialize it in the following sections. Note that the basic operation in it is compositional, not linear.

Let us reformulate and restrict the definition of GRID. The GRID (Growth as Random Iterated Diffeomorphisms) is a set of mappings on the space of diffeomorphisms $D I F F E O: \Xi \rightarrow X$. The set is generated by the elementary mappings

$$
\phi_{\text {radial }}(\eta): x(\xi) \rightarrow x^{\prime}(\xi), \xi \in \Xi
$$

with the property that

$$
\text { direction }\left[x^{\prime}(\xi)-\eta\right]=\operatorname{direction}[x(\xi)-\eta] .
$$

This means that the displacement vector $x^{\prime}-\eta$ is parallel to the vector $x-\eta$ itself: the cells grow or decay radially from the seed $\eta$. Actually we have used the form

$$
x \rightarrow(x-x(\eta)) \psi(\|x-x(\eta)\|),
$$

where the real-valued function of a real argument $\psi$ maps the interval $[0, \infty)$ diffeomorphically onto itself with 0 as a fixed point. This is called the radial version of GRID.

Then form the set

$$
\text { radialGRID }=\overline{\left[\phi_{\text {radial }}(\eta) ; \forall \eta \in \Xi\right]_{\circ}},
$$

where $[\cdot]_{\circ}$ means the algebraic closure with respect to function composition and the horizontal bar indicates topological closure with respect to some suitable topology on the set of diffeomorphisms. Then we have

Proposition. The set radialGRID forms a noncommutative topological subgroup of the diffeomorphic group. It is a proper subgroup.

5.1.1. Invariant case. We shall concentrate our study on situations where the diffeomorphisms $\phi^{\alpha}$ are invariant with respect to the translation group in $\mathbf{R}^{2}$. Then, there exists a diffeomorphism $\psi^{\alpha\left(\xi_{\text {seed }}, t\right)}$ such that

$$
\phi^{\alpha\left(\xi_{\text {seed }}, t\right)}=\xi_{\text {seed }}+\psi^{\alpha\left(\xi_{\text {seed }}, t\right)}\left(x(\xi)-x\left(\xi_{\text {seed }}\right)\right) .
$$

Note that the mapping $\psi$ depends on the nature of the seed, through $\alpha$, but not on its location. Let $d(x(\xi))$ denote the displacement undergone by the point $x(\xi)$; it is given by

$$
d(x(\xi))=\phi^{\alpha\left(\xi_{\text {seed }}, t\right)}(x(\xi))-x(\xi)=\psi^{\alpha\left(\xi_{\text {seed }}, t\right)}\left(x(\xi)-x\left(\xi_{\text {seed }}\right)\right)-\left[x(\xi)-x\left(\xi_{\text {seed }}\right)\right] .
$$

Setting $\theta^{\alpha\left(\xi_{\text {seed }}, t\right)}(u)=\psi^{\alpha\left(\xi_{\text {seed }}, t\right)}(u)-u$, we can write

$$
d(x(\xi))=\theta^{\alpha\left(\xi_{\text {seed }}, t\right)}\left(x(\xi)-x\left(\xi_{\text {seed }}\right)\right) .
$$


It is important to realize when using these relations that the map $x: \Xi \rightarrow X$ depends on time, it changes as the organism develops. To emphasize this we shall sometimes write $x(\xi, t)$.

5.1.2. $k$-functions for influence decay. This still leaves a lot of leeway in defining the diffeomorphisms. We shall look at one specification using the notation given in Figure 1 . We shall let the displacement away from the seed take place in the radial direction. Construct a unit vector $u_{s}$ in the $\xi_{2}$ direction at the seed $x\left(\xi_{\text {seed }}\right)$, and another unit vector $u=v /\|v\|$, where $v=x(\xi)-x\left(\xi_{\text {seed }}\right)$. With $c=\left\langle u, u_{s}\right\rangle$ expressing the cosine of $\tau$, the angle between $u$ and $u_{s}$, introduce a radial displacement function $k=k(c)$. The reason behind this choice is as follows. An area in the neighborhood of the seed, called the active area, is expanded (or contracted) at the rate $k ; k>1$ means expansion while $k<1$ means contraction. Outside the active area no change is made except to push layers away (or inwards) to compensate for the local expansion (contraction). This procedure constructs a diffeomorphism $\psi$ that is invariant to radial distance within the disk of radius $\delta$. In polar coordinates, with the origin at the seed and the angle measured from the $\xi_{1}$ axis, we will have transformations that can be written as

$$
(r, \theta) \rightarrow(k(\theta) r, \theta)
$$

so that the $k$ function regulates the local amount of growth/decay, with the value $k=1$ meaning no change. The dependence of $k(c)$ on $\tau$ shows how the size of the radial displacement is a function of $\tau$.

Several examples of the rate function $k$ are shown in Figure 7, plotted against $c$ (left panel) and $\tau$ (right panel). The left panel in each figure pair shows how some of the points of $\Omega$ move during this primitive deformation (or stay the same).

As stated earlier, the deformation specified by $k$ takes place only in the active area. Let the size of the active area be expressed through a small number called $\delta$, and let $v=x(\xi)-x\left(\xi_{\text {seed }}\right)$ be the relative location vector for point $x(\xi)$ at which the displacement is being computed. The vector $v$ makes an angle $\tau$ with the normal $u_{s}$ such that $c=\cos (\tau)$ determines the stretch factor $k(c)$, where $k$ is a rate function described above. That is, $c=\left\langle u_{s}, v\right\rangle /\|v\|$. Each component of the vector $v$ is transformed into the corresponding component of $\tilde{v}$ according to a function $\rho$, i.e. $\tilde{v}_{i}=\rho\left(v_{i}, c\right)$, where

$$
\rho(r, c)=\left\{\begin{array}{cc}
k(c) r & \text { if } \quad r<\delta \\
\operatorname{sign}(r)(k(c)-1) \delta+r & \text { if } r \geq \delta
\end{array}\right.
$$

In the coordinate system centered at $x\left(\xi_{\text {seed }}\right), \psi^{\alpha}$ defines a diffeomorphism according to $\psi^{\alpha}(v)=\tilde{v}$. Note that once the function $\rho(r, c)$ is defined and fixed, the rate function $k$ completely determines the diffeomorphism $\psi^{\alpha}$, and that $\psi^{\alpha}\left(\begin{array}{l}0 \\ 0\end{array}\right)=\left(\begin{array}{l}0 \\ 0\end{array}\right)$. In the fixed coordinate system, centered at the origin, the new location is given by $x(\xi)+d(x(\xi))$, where $d(x(\xi))=\tilde{v}-v$. A simple substitution verifies:

$$
\begin{aligned}
\phi^{\alpha}(x(\xi)) & =x(\xi)+d(x(\xi))=x(\xi)+\tilde{v}-v \\
& =x(\xi)+\psi^{\alpha}(v)-v=x\left(\xi_{\text {seed }}\right)+\psi^{\alpha}\left(x(\xi)-x\left(\xi_{\text {seed }}\right)\right) .
\end{aligned}
$$


$\psi^{\alpha}$ is a diffeomorphism that acts on vectors expressed in a coordinate system centered at $x\left(\xi_{\text {seed }}\right)$, while $\phi^{\alpha}$ is a diffeomorphism that acts on vectors expressed in the fixed coordinate system centered at the origin. $\theta^{\alpha}$ is the actual displacement in either coordinate system.

Summarizing the three diffeomorphisms with notation that will be used in the following, we have:

$$
\begin{aligned}
\psi^{\alpha}(v) & =\tilde{v} \\
\phi^{\alpha}(x(\xi)) & =x\left(\xi_{\text {seed }}\right)+\psi^{\alpha}\left(x(\xi)-x\left(\xi_{\text {seed }}\right)\right), \\
\theta^{\alpha}(v) & =\psi^{\alpha}(v)-v .
\end{aligned}
$$

While the synthesized growth behavior based on (5.13) works reasonably well it has a feature that is not desirable. Say that we place a growth generator seed $=$ uni-sourceforward in the $\Xi$-space. This results in growth not only around the seed but also closer to the boundary. To compensate for this behavior we introduce another $k$-function

$$
k(r, \phi)=r\left\{1+k(\phi) \exp \left[-d\left(\frac{r}{s t e p}\right)\right]\right\},
$$

where the function $d(\cdot)$ could be based on the $l_{2}$ metric

$$
d(x)=x_{1}^{2}+x_{2}^{2}, x=\left(x_{1}, x_{2}\right),
$$

or on the $l_{1}$ metric

$$
d(x)=\left|x_{1}\right|+\left|x_{2}\right|
$$

so that for small $r$, growth occurs with the rate $k(\phi)$ while for greater $r$, the push-out gradually decreases; see Figure 8 a. We show an example of the resulting deformation in Figure 8b.

Clearly the undesired effect close to the boundary $\partial \Xi$ has been eliminated. We therefore recommend the $k$-function in (5.14). The effect of growth on a vector $z=x-x_{\text {seed }}$ will then be

$$
z \rightarrow z^{\prime}=z\left\{1+k(\phi) \exp \left[-\left(\frac{\|z\|^{2}}{\text { step }}\right)^{2}\right]\right\}
$$

so that we get the growth increment

$$
\Delta z=z k(\phi) \exp \left[-\left(\frac{\|z\|^{2}}{s t e p}\right)^{2}\right] .
$$

For a later purpose we will compute the Jacobian of the transformation $\phi^{\alpha}(x)$. The Jacobian matrix is given by

Elements of $J$ are given by

$$
J=\left[\begin{array}{ll}
\frac{\partial \phi_{1}^{\alpha}}{\partial x_{1}} & \frac{\partial \phi_{2}^{\alpha}}{\partial x_{1}} \\
\frac{\partial \phi_{1}^{\alpha}}{\partial x_{2}} & \frac{\partial \phi_{2}^{\alpha}}{\partial x_{2}}
\end{array}\right]
$$

$$
\frac{\partial \phi_{i}^{\alpha}}{\partial x_{j}}=\left\{\begin{array}{cc}
0 & \text { if } i \neq j \\
k(c) & \text { if } i=j \text { and } x_{j}<\delta \\
1 & \text { if } i=j \text { and } x_{j} \geq \delta
\end{array}\right.
$$


Hence, the determinant of $J$ can take values $k(c)^{2}, k(c)$, and 1, depending upon whether none, one, or both components of $x(\xi)$ are close to components of $x\left(\xi_{\text {seed }}\right)$. In polar coordinates $(r, \theta)$, the resulting Jacobian $J$ can also be found as the ratio of the area elements $r \times d r \times d \tau$ and $k(\theta) r \times k(\theta) d r \times d \theta$.

5.1.3. Isotropic case. A special case of considerable interest is when the $k$-function does not depend upon the direction $\theta$. Then small circles will grow/decay into small circles; we have an isotropic situation. Note that the value of $k$ is constant with respect to changes in direction but may vary with the Darcyan coordinate $\xi$ so that aleomorphic growth can occur.

The set of diffeomorphisms generated by this radial form of GRID will be denoted radialGRID.

5.1.4. Are the mappings diffeomorphic? We must find conditions that ensure that the mappings are diffeomorphic. Consider the displacement of a point $x$ to the point

$$
\xi+\psi(\|v\|)(x-x(\xi)), v=x-x(\xi),
$$

where $\psi(\cdot)$ is a positive function. For what $\psi$-functions does this produce a diffeomorphism? If $\psi \equiv 1$ the mapping is of course just the identity $x \rightarrow x$.

Can it happen for two points $x \neq x^{\prime}$ that both of them are mapped to the same point $y$, so that $\xi+\psi(\|x-\xi\|)[x-\xi]=\xi+\psi\left(\left\|x^{\prime}-\xi\right\|\right)\left[x^{\prime}-\xi\right]$ ? Then $x-\xi \propto x^{\prime}-\xi$ so that the two difference vectors are collinear. On that line introduce a $1 \mathrm{D}$ coordinate $u$ with zero at the seed point. The mapping expressed in this coordinate is then

$$
x \rightarrow \xi+\psi(|u|) u \text {. }
$$

If we ask that the function on the right-hand side be increasing we get a bijective map. Hence

Lemma. If the $\psi$-function satisfies the inequality

$$
\psi^{\prime}(|u|) u \operatorname{sign}(u)+\psi(|u|)>0,
$$

then the mapping defined in (5.20) is diffeomorphic.

Applying this to the mapping with constant $k$ we see that it is indeed diffeomorphic since $\psi^{\prime}(u) \operatorname{sign}(u) u+\psi(u)=k>0$. Some $\psi$-functions require a constraint on the STEP variable.

5.2. Randomness in GRID. Since most biological phenomena growth is not completely deterministic, so it must be represented by probabilistic models. It is well known how to introduce a measure on a group, but here we are faced with the particular properties of the diffeomorphic group and how to exploit this for the construction of the measure.

We expect a large number of cell decisions, all of them of small effect, but together resulting in visible growth. This observation motivates the discussion of limit theorems in section 7.3. With some choice of area unit we shall denote the intensity of events by $\lambda(t, \xi)=\lambda(t) \times \lambda^{\alpha}(\xi)$ per area unit. Here $\lambda^{\alpha}$, expressing the gene controlled speed of events per $d x$ area unit will be kept fixed, while $\lambda(t)$ eventually will be made large: expressing the fact that we will be dealing with many events. Also, the area element should be $d x$ and not $d \xi$. Indeed, the cell density is expressed in $X$, having increased or decreased from the original form of the organism. At the same time the parameter step 
will be made to tend to zero: small range of growth (decay). With standard assumptions of independence and ruling out multiple events we are led to a Poisson process in Darcyan space-time of the seed points.

Introduce the cumulative diffeomorphisms

$$
\Phi_{n}=\phi_{n} \circ \phi_{n-1} \circ \ldots \circ \phi_{2} \circ \phi_{1}
$$

and the two maps $\Phi_{n+1}: x \mapsto y, \Phi_{n}: x \mapsto y$ given by the $\theta$ expressions in Section 5.1.2. In all situations we have met, the measures have been absolutely consitnuous with some densities, $p_{n+1}(y \mid x)$ and $p_{n}(y \mid x)$ respectively. Let us condition the probabilities by assuming that exactly one random seed is introduced from $n$ to $n+1$. For fixed $x \in \Omega(t)$ we ask how the conditional probability measures $P_{n+1}(y \mid x)$ and $P_{n}(y \mid x)$ are related. For some Borel set $F \subset \Omega(t)$ we have

$$
\begin{aligned}
P_{n+1}(F \mid x) & =\int_{x \in \Omega(t)} P[x+\theta(x-z) \in F] p_{n}(x) d x \\
& =\int_{x \in \Omega(t)} P\left[x-z \in \Psi_{n}^{-1}(F-x)\right] p_{n}(x) d x
\end{aligned}
$$

in terms of the inverse map $\Psi^{-1}$. But this can be written as

$$
\begin{array}{r}
\int_{x \in \Omega(t)} P\left[z \in x-\Psi_{n}^{-1}(F-x)\right] p_{n}(x) d x \\
=\int_{x \in \Omega(t)} \int_{z \in \Psi_{n}^{-1}(F-x)} p_{n}(x) d x f(z) d z
\end{array}
$$

with the normalized density $f(z) \propto \lambda(z)$. Hence we get the density

$$
p_{n+1}(y \mid x)=\int_{x \in \Omega(t)} p_{n}(x-z \mid x) f[\Psi(-1)(y-x)] J_{\theta}^{-1} d x
$$

with the Jacobian $J_{\theta}=\frac{\partial \theta}{\partial x}$. Thus we have proved the

Proposition. The densities $p_{n+1}$ and $p_{n}$ are related by the operator

$$
(T p)(y)=\int_{z} p[x-z] f\left[\Psi^{-1}(y-x)\right] J_{\theta}^{-1} d x .
$$

5.3. Thermodynamic limit. We shall use an analogy with statistical mechanics. The quantities in statistical mechanics are random in nature, but the thermodynamic limit involves nonrandom variables. This is due to a limit process such as the law of large numbers. For fixed $\xi_{\text {seed }}$ the instantaneous growth is given by the important equation

$$
\frac{\partial x(\xi, t)}{\partial t}=\theta_{\xi}^{\alpha(t)}\left[x(\xi, t)-x\left(\xi_{\text {seed }}, t\right)\right]
$$

where $\xi_{\text {seed }}$ is a random variable induced by a Poisson point process and the $\theta$ function was introduced in Section 5.1.2. Hence we get the thermodynamic limit

$$
\frac{\partial x(\xi, t)}{\partial t}=\sum_{\alpha=1}^{A} \int_{\xi_{\text {seed }} \in \Xi} \theta_{\xi}^{\alpha(t)}\left[x(\xi, t)-x\left(\xi_{\text {seed }}, t\right)\right] P\left(d \xi_{\text {seed }}, \alpha ; t\right) .
$$


In (5.28) we could substitute, for example,

$$
\theta(z)=z k(\phi) \exp \left(-\|z\|^{2} / \text { step }^{2}\right) .
$$

The thermodynamic limit represents "typical" growth and neglects the random biological variability: it integrates out the randomness. Returning to (5.27) and solving it over a time interval $\left(t_{1}, t_{2}\right)$ so short that $x(\xi, t)$ does not have time to change much, we get the growth increment

$$
\begin{aligned}
\Delta x\left(\xi, t_{1}\right) & =x\left(\xi, t_{2}\right)-x\left(\xi, t_{1}\right) \\
& \approx\left(t_{2}-t_{1}\right) \sum_{i=1}^{n} \theta_{\xi}^{\alpha\left(\eta_{i}, t_{1}\right)}\left[x\left(\xi, t_{1}\right)-x\left(\eta_{i}, t_{1}\right)\right],
\end{aligned}
$$

where the $\eta$ 's are the random seeds activated during the short time interval distributed as a Poisson process with the intensity $\Lambda=\lambda \times \lambda^{\alpha\left(\cdot, t_{1}\right)}$. This can be written as

$$
\Delta x\left(\xi, t_{1}\right) \approx\left(t_{2}-t_{1}\right) \int_{\eta \in \Xi} \theta_{\xi}^{\alpha\left(\eta, t_{1}\right)}\left[x\left(\xi, t_{1}\right)-x\left(\eta, t_{1}\right)\right] F^{*}(d \eta),
$$

where $F^{*}$ is the empirical measure of the realization of the Poisson process putting a unit mass at each of the seed points.

Arguing heuristically we then get

$$
\Delta x\left(\xi, t_{1}\right) \approx\left(t_{2}-t_{1}\right) \lambda \times \int_{\eta \in \Xi} \theta_{\xi}^{\alpha\left(\eta, t_{1}\right)}\left[x\left(\xi, t_{1}\right)-x\left(\eta, t_{1}\right)\right] F(d \eta) ; \quad F=E F^{*},
$$

an approximation that may be useful in the analytical study of the GRID model.

5.4. Some illustrations of GRID. A Power Point presentation can be found on the website www.dam.brown.edu/ptg under PUBLICATIONS 2005; it has some film clips showing pattern synthesis of GRID growth.

\section{Analytical study of GRID.}

6.1. Completeness properties of the GRID representation. Of course the space radialGRID $(X) \subseteq D I F F E O(X)$, but is it a proper subset in the sense that some diffeomorphisms on $X$ cannot be generated by radialGrid? Let us study this question for the thermodynamic version for a given $x(\xi, t)$ and $t$. To solve this problem we shall consider all the velocity fields

$$
v=\sum_{i=1}^{m}\left(x-x\left(\eta_{j}\right)\right) \psi\left(\left\|x-x\left(\eta_{j}\right)\right\|^{2} a_{j}, \quad \eta_{j} \in \Xi\right.
$$

belonging to the algebraic closure $[\psi]_{o}$. But for $\psi(r)=\frac{\partial \Psi(r)}{\partial r}$ we can write the components of $v$ as

$$
v_{1}=\frac{1}{2} \frac{\partial \Psi}{\partial x_{1}}, \quad v_{2}=\frac{1}{2} \frac{\partial \Psi}{\partial x_{2}}
$$

or in vector form

$$
v=\frac{1}{2} \operatorname{grad} \Psi
$$

so that the velocity field is obtained from a velocity potential $\Psi$. But that means that the field is irrotational. 
On the other hand, say that the field $v$ is irrotational so that there exists a scalarvalued function $\Psi$ for which $v=-\operatorname{grad} \Psi$. Now let us form linear combinations

$$
l(x)=\sum_{j=1}^{m} c_{j} \Psi\left(\left\|x-x\left(\eta_{j}\right)\right\|\right), \quad \eta_{j} \in \Xi .
$$

But since $x(\cdot, t)$ maps $\xi$ bijectively onto $X$ we can write this as

$$
l(x)=\sum_{j=1}^{m} c_{j} \Psi\left(\left\|x-x^{j}\right\|\right), \quad x^{j} \in X .
$$

Such linear combinations are dense in $L_{2}(X)$ if the Fourier transform of $\psi$ does not vanish for real arguments; see Wiener (1933). We have

$$
\bar{\psi}(z)=\int_{-\infty}^{\infty} \exp (i\langle z, x\rangle) \Psi(\|x\|) d x \neq 0, \quad \forall z \in \mathbf{R} .
$$

Changing to polar coordinates

$$
z=(\rho \cos \theta, \rho \sin \theta), \quad x=(r \cos \alpha, r \sin \alpha),
$$

the integral can be written as

$$
\begin{aligned}
\bar{\psi}(z) & =\int_{r=-\infty}^{\infty} \int_{\alpha=0}^{2 \pi} \exp [i r \cos (\theta-\alpha)] \Psi(r) r d r d \theta \\
& =2 \pi \int_{r=0}^{\infty} J_{0}(r) \Psi(r) r d r .
\end{aligned}
$$

If this completeness condition is satisfied we can approximate the velocity potential $V$ by linear combinations so that after forming partial derivatives with respect to $x_{1}, x_{2}$ we get a linear form similar to (6.4). Hence we have proved the

Proposition. If the Hankel transform

$$
\int_{r=0}^{\infty} J_{0}(r) \Psi(r) r d r
$$

does not vanish for real arguments, we have the completeness result

$$
\overline{[\text { radialGRID }]_{o}}=\text { irrotationalDIFFEO }(X) \text {. }
$$

In the special case $\psi(r)=\exp \left(-r^{2} /\right.$ step $\left.^{2}\right)$ the condition is satisfied so that completeness holds. The next question is naturally what modification of GRID is needed to represent velocity fields with nonvanishing curl. One way of achieving this is to replace (6.1) by the linear combinations

$$
\begin{aligned}
& v_{1}=\sum_{j=1}^{m}\left(x_{1}-x_{1}^{1, j}\right) \psi\left(\left\|\left(x-x^{1, j}\right)\right\|\right) a_{j}^{1} ; \\
& v_{2}=\sum_{j=1}^{m}\left(x_{2}-x_{2}^{2, j}\right) \psi\left(\left\|\left(x-x^{2, j} j\right)\right\|\right) a_{j}^{2} .
\end{aligned}
$$

Reasoning as above we get completeness for this version EXT extending GRID:

$$
\overline{[E X T]_{o}}=\operatorname{DIFFEO}(X) \text {. }
$$


But note that (6.11) violates the principle of biological relativity: the growth decisions depend upon absolute coordinates, not just on Darcyan ones. Hence the modified growth model does not belong to the version of GRID studied above.

REMARK. It is natural to use algebraic closure by composition for the random (Poisson) form of GRID and linear combinations for the thermodynamic version.

6.2. Flow type produced by GRID. Is it possible to characterize in general terms the flows that GRID produces? We shall consider the version described in Section 5.2.2 with radial displacements. We shall need some elementary relations for the mapping $x \rightarrow y$ :

$$
\begin{aligned}
\frac{\partial y_{1}}{\partial x_{1}} & =\frac{\partial y_{1}}{\partial r} \frac{\partial r}{\partial x_{1}}+\frac{\partial y_{1}}{\partial \theta} \frac{\partial \theta}{\partial x_{1}}=\frac{\partial y_{1}}{\partial r} \frac{x_{1}}{r}-\frac{\partial y_{1}}{\partial \theta} \frac{x_{2}}{r^{2}} \\
& =\frac{\partial y_{1}}{\partial r} \cos \theta-\frac{\partial y_{1}}{\partial \theta} \sin \theta / r \\
\frac{\partial y_{1}}{\partial x_{2}} & =\frac{\partial y_{1}}{\partial r} \frac{\partial r}{\partial x_{2}}+\frac{\partial y_{1}}{\partial \theta} \frac{\partial \theta}{\partial x_{2}} \\
& =\frac{\partial y_{1}}{\partial r} \frac{x_{2}}{r}+\frac{\partial y_{1}}{\partial \theta} \frac{x_{1}}{r^{2}}=\frac{\partial y_{1}}{\partial r} \sin \theta+\frac{\partial y_{1}}{\partial \theta} \cos \theta / r \\
\frac{\partial y_{2}}{\partial x_{1}} & =\frac{\partial y_{2}}{\partial r} \frac{\partial r}{\partial x_{1}}+\frac{\partial y_{2}}{\partial \theta} \frac{\partial \theta}{\partial x_{1}} \\
& =\frac{\partial y_{2}}{\partial r} \frac{x_{1}}{r}-\frac{\partial y_{2}}{\partial \theta} \frac{x_{2}}{r^{2}}=\frac{\partial y_{2}}{\partial r} \cos \theta-\frac{\partial y_{2}}{\partial \theta} \sin \theta / r \\
\frac{\partial y_{2}}{\partial x_{2}} & =\frac{\partial y_{2}}{\partial r} \frac{\partial r}{\partial x_{2}}+\frac{\partial y_{2}}{\partial \theta} \frac{\partial \theta}{\partial x_{2}} \\
& =\frac{\partial y_{2}}{\partial r} \frac{x_{2}}{r}+\frac{\partial y_{2}}{\partial \theta} \frac{x_{1}}{r^{2}}=\frac{\partial y_{2}}{\partial r} \sin \theta+\frac{\partial y_{2}}{\partial \theta} \cos \theta / r .
\end{aligned}
$$

Also

$$
\begin{aligned}
\frac{\partial y_{1}}{\partial r} & =\frac{\partial k}{\partial r} \cos \theta \\
\frac{\partial y_{1}}{\partial \theta} & =\frac{\partial k}{\partial \theta} \cos \theta-k \sin \theta \\
\frac{\partial y_{2}}{\partial r} & =\frac{\partial k}{\partial r} \sin \theta \\
\frac{\partial y_{2}}{\partial \theta} & =\frac{\partial k}{\partial \theta} \sin \theta+k \cos \theta
\end{aligned}
$$

Combining equations (6.13)-(6.16) with (6.17)-(6.20) we get the divergence of the growth as

$$
\frac{\partial y_{1}}{\partial x_{1}}+\frac{\partial y_{2}}{\partial x_{2}}-2=\frac{\partial k}{\partial r}+k / r-2
$$

To characterize the rotational properties of the flow we compute the curl and get after some calculation

$$
\operatorname{curl}=\frac{\partial y_{1}}{\partial x_{2}}-1-\frac{\partial y_{2}}{\partial x_{1}}+1=\frac{1}{r} \frac{\partial k}{\partial \theta}
$$


Summing up we have

Proposition. For the GRID model with radial growth the flow is characterized by

$$
\begin{gathered}
\operatorname{div} v=\frac{\partial k}{\partial r}+k / r-2, \\
\operatorname{curl} \frac{1}{r} \frac{\partial k}{\partial \theta} .
\end{gathered}
$$

In the special case when $k=K r$ with $K$ independent of $r$, which typically happens in the neighborhood of the seed, the divergence becomes

$$
\operatorname{div} v=2(K-1)
$$

of course, this is positive (expansion) if $K>1$ and negative (compression) when $K<1$.

In the isotropic case the flow is irrotational, so that the velocity field can be expressed in terms of a velocity potential $E$ :

$$
v=\operatorname{grad} E .
$$

7. A calculus of growth and patterns of growth. After these considerations we are ready to formalize our ideas concerning biological growth in the form of a calculus: a Darcyan calculus to emphasize the fact that all the biological decisions are carried out in a Darcyan $\xi$-coordinate system.

7.1. Generators of growth. Following the pattern-theoretic routine, see Grenander (1993), Chapter 1 , we introduce a generator space $G_{\text {prim }}$ of growth primitives. A generator $g \in G_{\text {prim }}$ will have the form

$$
g=\langle\Delta T|\Delta \Xi| k\rangle,
$$

where

1) $\Delta T=[0,1)$ is the standardized time interval of growth when the generator is applied;

2) $\Delta \Xi \subset \Xi$ is the subset of the Darcyan coordinate system;

3) $k$ is the $k$-function introduced earlier.

7.2. Similarity groups for growth. The similarity group will be a product

$$
S=S^{T} \times S^{\Xi} \times S^{a m p l} \times S^{\text {angle }}
$$

with

1) $S^{T}=\mathrm{SA}(1)$, applying an affine one-dimensional transformation $(a, b): t \rightarrow a+b t$ to $T$ so that the standard time interval $[0,1)$ is transformed to $\left[t_{1}, t_{2}\right)$;

2) $S^{\Xi}=\operatorname{DIFFEO}(\Xi)$ applied to subsets of $\Xi$;

3) $S^{a m p}=\mathbf{R}_{+}^{1}$ applied to the values of the $k$-function;

4) $S^{\text {angle }}=\mathbf{T}^{1}$ applied to the direction of the $k$-function. 
7.3. Configurations of growth. Using these generators and group elements we first extend the generator space algebraically to $G=S G_{\text {prim }}$ and then create configurations $c=\sigma\left(s_{1} g_{1}, s_{2} g_{2}, \ldots, s_{n} g_{n}\right)$ that can take the form in the example.

\begin{tabular}{|l|l|l|} 
group elements & subset & $k$-function \\
\hline$\langle(1,3), i d, 2,0\rangle$ & $\Xi_{1}$ & $\langle$ unipolar - source - midwards $\rangle$ \\
$\langle(4,2), i d, 1,0\rangle$ & $\Xi_{2}$ & $\langle$ unipolar - sink - forwards $\rangle$ \\
$\langle(6,2), i d, 3,0\rangle$ & $\Xi_{3}$ & $\langle$ bipolar - source - midwards - sink - backwards $\rangle$
\end{tabular}

8. GRID analysis. The GRID representation presents many analytical problems, and we shall study a few of them here.

8.1. Growth guides. Let us first study the special case when we have a single $\alpha$-value and $\lambda^{\alpha}$ is constant in time. Then the thermodynamic limit can be written as

$$
\frac{\partial x(\xi, t)}{\partial t}=\int_{\xi_{\text {seed }} \in \Xi} \theta\left[x(\xi, t)-x\left(\xi_{\text {seed }}, t\right)\right] F\left(d \xi_{\text {seed }}\right), \quad \theta(u)=\psi(u)-u,
$$

where $F$ is a bounded measure, not necessarily normalized to total variation one, and we can formulate the inference problem in variational form

$$
i n f_{F} \int_{\xi \in \Xi}\left\{I_{1}[x(\xi, 0)]-I_{2}[x(\xi, T)]\right\}^{2} \frac{m(d x)}{d \xi} d \xi
$$

when the organism observed at $t=0$ is $I_{1}$ and at $t=T$ has become $I_{2}$. Furthermore, $m$ stands for Lebesgue measure in $X$ while $d \xi$ means Lebesgue measure in the Darcyan space $\Xi$. The Radon-Nikodým derivative $\frac{m(d x)}{d \xi}$ is needed in order to relate growth changes to the $X$ coordinate system.

Actually, the practical version of (8.2) is when the growth measure $F$ consists of a finite number $m$ of atoms so that growth is governed by an autonomous system of functional differential equations

$$
\frac{\partial x(\xi, t)}{\partial t}=\sum_{j=1}^{m} \theta\left[x(\xi, t)-x\left(\xi_{s e e d}^{j}, t\right)\right] F_{j}
$$

Choosing $\xi=\xi_{\text {seed }}^{j}$ we get the ODE

$$
\frac{\partial y_{i}(t)}{\partial t}=\sum_{j=1}^{m} \theta\left[y_{i}(t)-y_{j}(t)\right] F_{j}
$$

with $y_{i}(t)=x\left(\xi_{i}, t\right)$. The trajectories of the $y_{i}$ 's, the shape guides, may be biologically meaningful. The guides can have different $k$ values, which means a minor modification of (5.14). The guide polarities, i.e., the signs of the $k_{j}$, will be denoted + and o in the graphics. An example of the growth guide system is shown in Figure 9.

The figure shows that the behavior of the resulting growth guides is not intuitive. The seeds themselves will not move in isolation, only due to the influence of seeds sufficiently close to them. This is a reflection of the fact that biological entities do not live in absolute space, only in the Darcyan generated by the DNA distribution of seeds in the organisms. 
Returning to equation (8.3), it can be written in terms of the shape guides as

$$
\frac{\partial x(\xi, t)}{\partial t}=\sum_{j=1}^{m} \theta\left[x(\xi, t)-y_{j}(t)\right] F_{j}
$$

in other words, as a nonautonomous ordinary differential equation in $x(\xi, t)$ for each $\xi \in \Xi$. Note that the relation between the shape directors does not depend upon $x(\xi, t)$ for other $\xi$ 's but upon the seeds.

8.2. Minimum attained. Let $F_{1}, F_{2}, F_{3}, \ldots$ be a sequence leading to the inf in (8.2) and get $F_{i}=$ TotalVariation $\left(F_{i}\right) \times P_{i}$ with the measures $P_{i}$ having total variation 1 . Then, appealing to tightness, there is a weakly convergent subsequence $P_{i_{1}}, P_{i_{2}}, P_{i_{3}}, \ldots \rightarrow P_{\text {lim }}$, so that

$$
\int_{\xi_{\text {seed }} \in \Xi} \theta\left[x(\xi, t)-x\left(\xi_{\text {seed }}, t\right)\right] F_{i_{\nu}}\left(d \xi_{\text {seed }}\right) \sim \text { TotalVariation }\left(F_{i_{\nu}}\right) \times h(\xi), \nu \rightarrow \infty,
$$

with

$$
h(\xi)=\int_{\xi_{\text {seed }} \in \Xi} \theta\left[x(\xi, t)-x\left(\xi_{\text {seed }}, t\right)\right] F_{\text {lim }}\left(d \xi_{\text {seed }}\right) .
$$

Hence the sequence TotalVariation $\left(F_{i_{\nu}}\right)$ must be bounded in order to make (8.6) small, so that we have compactness and thus the following.

Lemma. The inf is attained.

We do not believe that the minimum solution is unique in general. For the special case considered in this section, however, it is believed that the minimum is uniquely attained.

8.3. Limit theorems for GRID. With some further specification we shall now consider the following setup. The same mapping but for translations will be assumed, so we can leave out, for the moment, the $\alpha$ :

$$
x \rightarrow y=\frac{1}{\lambda} \phi\left[\left(x-x_{\text {seed }}\right) \lambda\right], \quad x, x_{\text {seed }} \in \mathbf{R}^{2},
$$

where $\phi(\cdot)$ reduces to translation at some finite distance from 0 so that its Jacobian $j$ is then equal to one. We plan to use the results of the following analysis for short intervals of growth so that $\alpha$ can be considered time independent. The parameter $\lambda$, see section 3 , is both a Poisson and a scale parameter that should be made to tend to infinity. Consider the random iterated diffeomorphism

$$
\begin{aligned}
& \phi^{(\lambda)}(x)=\frac{1}{\lambda} \phi\left[\left(x-x_{\text {seed }}^{(n)}\right) \lambda\right] \circ \frac{1}{\lambda} \phi\left[\left(x-x_{\text {seed }}^{(n-1)}\right) \lambda\right] \\
& \circ \ldots \circ \frac{1}{\lambda} \phi\left[\left(x-x_{\text {seed }}^{(1)}\right) \lambda\right],
\end{aligned}
$$

where the seeds $x_{\text {seed }}^{(i)}$ are Poisson points generated with the intensity $\lambda \times \lambda^{\alpha}$, with $\alpha$ fixed for the moment; $n$ will be a Poisson distributed random variable.

In the present section we shall deal with growth over a time interval $(t, t+h)$, where $h$ is small. In the mouse studies $h$ could be a week or less.

In terms of the partial derivatives of $\phi=\left(\phi_{1}, \phi_{2}\right)$,

$$
\phi_{i j}=\frac{\partial \phi_{i}}{\partial x_{j}},
$$


the Jacobian of the transformation in (8.8) takes the form

$$
\begin{aligned}
j=\frac{\partial(y)}{\partial(x)} & =\frac{1}{\lambda} \operatorname{det}\left(\begin{array}{cc}
\phi_{11}\left[x-x_{\text {seed }}\right] \lambda & \phi_{12}\left[x-x_{\text {seed }}\right] \lambda \\
\phi_{21}\left[x-x_{\text {seed }}\right] \lambda & \phi_{22}\left[x-x_{\text {seed }}\right] \lambda
\end{array}\right) \\
& =\lambda \operatorname{det}\left(\begin{array}{cc}
\phi_{11}\left[x-x_{\text {seed }}\right] & \phi_{12}\left[x-x_{\text {seed }}\right] \\
\phi_{21}\left[x-x_{\text {seed }}\right] & \phi_{22}\left[x-x_{\text {seed }}\right]
\end{array}\right) .
\end{aligned}
$$

For large $\|x\|$ the last determinant in (8.11) becomes 1 , so that $j$ is also 1 for large $x$-values.

For use below we introduce the Fourier transform

$$
\Phi(u)=\int \exp [i\langle u, x\rangle] \log [j(x)] d x,
$$

noting that the integrand has finite support so that the integral is well defined.

But the Jacobian of the transformation (8.9) is with the straightforward choice of notation

$$
J^{\lambda}(x)=\prod_{i=1}^{n} j^{i}\left(x^{i}-x_{\text {seed }}^{i}\right)
$$

where the iterations yield $x \rightarrow x^{1} \rightarrow x^{2}, \ldots \rightarrow x^{n}$ so that

$$
\log J^{\lambda}(x)=\sum_{i=1}^{n} \log \left[j^{i}\left(x^{i}-x_{\text {seed }}\right)\right]
$$

In the case of a small duration $h$ we shall approximate

$$
\log J^{\lambda}(x) \approx \sum_{i=1}^{n} \log \left[j^{i}\left(x-x_{\text {seed }}\right)\right]
$$

since the $x$ 's have not had the time to change much.

To find the asymptotics of the random entity in (8.15) we shall apply a time-honored technique. As $\lambda \rightarrow \infty$ we have

$$
\frac{n}{\lambda} \stackrel{\text { in probability }}{\rightarrow} \Lambda=\int \lambda^{\alpha}(x) d x
$$

On the other hand, conditioned by fixing $n$, the $x_{\text {seed }}$ points are iid with a probability density

$$
p(x)=\frac{\lambda^{\alpha}(x)}{\int \lambda^{\alpha}(x) d x} .
$$

Combining this with the convergence of empirical distributions we get

ThEOREM 8.1. The GRID model satisfies, for short durations, the limit relation

$$
\lim _{\lambda \rightarrow \infty} \frac{1}{\lambda} \log J^{\lambda}(x) \approx \Lambda \int \log [j(x-z)] \lambda(z) d z .
$$

To estimate the growth parameter $\lambda(\cdot)$ we first estimate $J^{\lambda}(\cdot)$ in the obvious way from data. We then solve the integral equation (8.18) for the function $\lambda(\cdot)$. But this is not the whole truth. Indeed, in order to be able to guarantee consistent estimation as $\lambda \rightarrow \infty$ we should make sure that the integral equation cannot have more than one solution, so that, 
if $\lambda_{1}(\cdot)$ and a different $\lambda_{2}(\cdot)$ both satisfy $(8.18)$, then there exists a nontrivial solution $l(\cdot)$ of

$$
\int \log [j(x-z)] l(z) d z \equiv 0
$$

But this is related to a celebrated completeness result, see Wiener (1933), and has no nontrivial solution if the Fourier transform introduced in (8.12) does not vanish, $\Phi(u) \neq$ $0, \forall u \in \mathbf{R}^{2}$.

It is tempting to try estimating $\lambda(\cdot)$ by Fourier inversion, but we know from earlier experience that such procedures are inherently numerically unstable, so more direct methods are indicated. For example, let us consider the case studied in Section 2. Then the Jacobian is equal to the $k$-value in a small neighborhood disk $N=\left\{X:\left\|x-x_{\text {seed }}\right\|<\right.$ $S T E P\}$ and zero outside. But the Fourier transform $\Phi(u)$ is then

$$
\begin{array}{rl}
2 k^{2} \int_{r=0}^{S T E P} \exp [i r\|u\| \cos \theta] r d r d \theta=2 k^{2} \pi \int_{r=0}^{S T E P} & r J_{0}(\|u\|) d r \\
& =2 k^{2} \pi S T E P J_{1}(S T E P\|u\|)
\end{array}
$$

in terms of Bessel functions. But it is known that $J_{1}(\cdot)$ has positive real zeros $0<$ $z_{1}<z_{2}<\ldots$ so that we cannot guarantee consistent estimation. Indeed, we can add to any estimate obtained as described a function belonging to the subspace $\mathcal{F}$ obtained by closing algebraically and topologically the family of trigonometric functions

$$
\left\{\exp \left[i\left\langle x, u_{k}\right\rangle\right] ;\left\|u_{k}\right\|=z_{k} / S T E P ; k=1,2, \ldots\right\} .
$$

The smallest wave numbers have $\left\|u_{\text {min }}\right\|=z_{1} / S T E P=3.8317 / S T E P$. If STEP is of the order of the action radius of cell decisions, then it follows that the lack of uniqueness has no practical implication.

The iterated form of equation (8.9) leads naturally to the question of whether or not there are probabilistic limit theorems in the stochastic diffeomorphic group that we introduced: either an LLN or a CLT. We shall study this question for the invariant case with a $k$-function as in Section 5.1.2 and consider the possible form of a CLT.

This should indeed be possible since the resulting deformation $d_{n}$ of the point $x(\xi)$ after $n$ (a random number!) of seeds is of the additive form

$$
d_{n}=\theta^{\alpha\left(\xi_{\text {seed }}^{n}, t_{n}\right)}\left(x\left(\xi, t_{n}\right)-x\left(\xi_{\text {seed }}^{n}, t_{n}\right)\right)+\ldots+\theta^{\alpha\left(\xi_{\text {seed }}^{1}, t_{1}\right)}\left(x\left(\xi, t_{1}\right)-x\left(\xi_{\text {seed }}, t_{1}\right)\right) .
$$

The simple additive form of (8.22) is due to the fact that we have exploited the translation invariance; otherwise, we would meet extra difficulties.

The general term in (8.22) is

$$
V_{i}=\theta^{\alpha\left(\xi_{\text {seed }}^{i}, t_{i}\right)}\left(x\left(\xi, t_{i}\right)-x\left(\xi_{\text {seed }}, t_{i}\right)\right),
$$

but it is important to realize that they are not stochastically independent since the $x(\xi, t)$ develop during the life of the organism. On the other hand, a simplification occurs such that in the total displacement the Darcyan coordinate $\xi$ occurs alone, not the neighboring values. Of course the growth is influenced by what happens elsewhere due to the influence of the seeds, but the dependence is only directly via a single $\xi$. We can think of this as individual growth and will sometimes leave out $\xi$ in the notation when it is fixed. 
Unfortunately we do not yet have any rigorous treatment of this problem and can only offer some heuristics, hoping that some analytically inclined colleague will be able to cover the logical holes in our discussion.

But first we characterize the local properties of the $s^{\alpha}$ in 2D form via the Jacobian matrix

$$
\mathbf{J}=\frac{\partial\left(x_{1}^{\alpha}, x_{2}^{\alpha}\right)}{\partial\left(\xi_{1}, \xi_{2}\right)}=\left(\begin{array}{ll}
\frac{\partial x_{1}^{\alpha}}{\partial \xi_{1}} & \frac{\partial x_{1}^{\alpha}}{\partial \xi_{2}} \\
\frac{\partial x_{2}^{\alpha}}{\partial \xi_{1}} & \frac{\partial x_{2}}{\partial \xi_{2}}
\end{array}\right)
$$

and write it in polar form as the product of a nonnegative matrix $P$ and an orthogonal matrix $O$ :

$$
\mathbf{J}=P O=\left(\begin{array}{cc}
\lambda_{1} & 0 \\
0 & \lambda_{2}
\end{array}\right)\left(\begin{array}{cc}
\cos \phi & \sin \phi \\
-\cos \phi & \cos \phi
\end{array}\right)
$$

We can then separate cases:

1) if $\lambda_{1}>1, \lambda_{2}>1$ : proper growth;

2) if $\lambda_{1}<1, \lambda_{2}<1$ : proper decay;

3) else: mixed development.

We shall exploit the fact that the Jacobian $J(x)$ is a multiplicative functional over the diffeomorphic group, i.e.,

$$
J\left(\phi_{1} \circ \phi_{2}\right)=J\left(\phi_{1}\right) J\left(\phi_{2}\right),
$$

and we shall consider the development of the Jacobian for fixed Darcyan $\xi$ when time $t$ increases.

The development process is Markovian, and we shall now derive expressions for the infinitesimal mean vector and covariance matrix for the process $J(t)$ that appear in Kolmogorov's forward equation. Consider the change over the short time interval $(t, t+h)$ conditioned by fixing $x(t)$ :

$$
\Delta x=x(t+h)-x(t)=0 \quad \text { with } \quad \text { probability } \quad 1-\lambda \times \lambda^{\alpha} h+o(h)
$$

and

$$
\Delta x=\theta^{\alpha\left(\xi_{\text {seed }}, t\right)}(x(t-x \text { seed })) \quad \text { with } \quad \text { probability } \quad \lambda \times \lambda^{\alpha} h+o(h)
$$

and other possibilities with probability $o(h)$. Using the broken linear form of the $k$ function discussed earlier we get the conditional expectation for the Jacobian of the displacement $x(t+h)-x(t)$ :

$$
h \times \lambda \times \lambda^{\alpha} \times \int_{S} k^{2}\left[\alpha\left(\xi_{\text {seed }}, t\right), c\right] P\left(d \xi_{\text {seed }}\right)+o(h)
$$

with the probability measure $P$ for the random seed $x_{\text {seed }}$ and for $\alpha(\xi, t)$. Integration is over the set $S$,

$$
S=\left\{\left\|x(t)-x_{\text {seed }}\right\|<S T E P\right\} .
$$

Now let $\lambda \rightarrow \infty$ and STEP $\downarrow 0$ in such a way that $\lambda \times S T E P^{2}=\Lambda$. We get the infinitesimal mean value vector

$$
\lambda^{\alpha} \Lambda \bar{k}\left[\alpha\left(\xi_{\text {seed }}, t\right), c\right] J(t) .
$$


The bar means averaging over the variable $c$. In other words, the infinitesimal mean value vector for the $J$ process is proportional to $J$.

For the infinitesimal variance matrix we get, with a similar argument, proportionality to $J^{2}$ so that the process is governed by an Ito stochastic differential equation of the form

$$
d y=a y d t+b y W(d t)
$$

known as the Geometric Brownian Motion. It corresponds to Kolmogorov's forward equation with the above infinitesimal means and variances. It follows that

Proposition. The iterated random diffeomorphism growth model leads to asymptotically lognormal distributions for the Jacobian process.

REMARK. The Jacobian contains only one-dimensional information while our current setup is in the plane. We believe it to be possible to get two-dimensional information by replacing the Jacobian determinant by the Jacobian matrix for the mapping $x(:)=$ $x(:, t) \rightarrow x(:, t+h)=y(:)$,

$$
M=\left[\begin{array}{ll}
\frac{\partial y_{1}}{\partial x_{1}} & \frac{\partial y_{1}}{\partial x_{2}} \\
\frac{\partial y_{2}}{\partial x_{1}} & \frac{\partial y_{2}}{\partial x_{2}}
\end{array}\right],
$$

and by deriving a 4D analog to the Geometric Brownian Motion for the four entries in $M$. The infinitesimal means and variances determine a Kolmogorov forward equation that will be a parabolic partial differential equation with one time variable and four space variables. This seems to be related to the CLT over the stochastic groups SL(2) and $\mathrm{SA}(2)$.

8.4. Analysis of simulated data. To get some feeling for how the theorem works we have simulated it and obtained the histograms for the Jacobians shown in Figure 10, The three histograms on the left are three different cases of Jacobian distributions while the three histograms on the right are distributions of $\log J$. The distribution function after a normal transformation is shown further to the right, both for $J$ and $\log J$. The agreement with the log normal hypothesis is good.

In Figure 11 we see a histogram for $J$ and of $\log J$ to the right for another simulation. The two rightmost panels show the normal transformation plots for $J$ on the left and for $\log J$ on the right. The agreement is good up to the 95 th percentile but not further out in the tail of the distribution. We make the reservation, however, that the limited agreement is with the proposition under the assumption of the GRID model; it does not say anything about agreement with real data; that remains to be seen.

It will be instructive to see how the behavior of $J$ depends upon the type of $k$-function. In the growth mode uni-source-forwards we get growth in the radial direction; compare the right panel of $\log J$ with the color bar in Figure 12. In the growth mode isotropicsource, isotropic-sink we get, see Figure 13, decay in blue and growth in red. The 
interesting growth mode uni-source-forwards, bi-source-forwards-sink-backwards results in Figure 14 with its more complicated development. These figures show clearly that the growth rates are directly related to the field of log Jacobian. The relation is, however, only qualitative, and it remains to find more detailed, quantitative equations for the relationship.

9. Inference. Having acquired images of $\Omega$, the question arises how they can be used to infer properties of the growth. We are going to study the following topics.

1) If the map $\phi$ is available, how can we derive properties of the underlying GRID structure? For example, how can we estimate the growth power measure $A$ or its density $a ?$

2) Given one or several observed images $I^{\mathcal{D}}$, how can this be exploited in order to estimate the biological mapping $\phi$ ? There are several versions of this question depending upon what group cascade is assumed to have generated the observations, for example a registration group, a photometric group and so on.

3) Starting from an anatomical atlas describing the architecture of $\Omega$ in terms of sub-organisms $\omega_{i}$, how can we automatically locate a given anatomical component, for example the hippocampus, in $I^{\mathcal{D}}$ ?

9.1. Estimate growth parameters when mapping given.

9.1.1. A special estimate of growth power. To start the discussion of inferences for GRID we shall introduce a method that is highly specialized but will help in setting the scope of the discourse. Given the deformation generated by the thermodynamic limit we want to estimate the density of the growth power measure, here denoted by $f=F^{\prime}$. Let us do this for the case when the rate in formula (5.14) is give as $d(x)=\left|x_{1}\right|+\left|x_{2}\right|$ and with isotropy, $k \equiv$ constant over direction. To see the problem clearly let us start with the $1 \mathrm{D}$ case so that the deformation becomes in the thermodynamic limit

$$
D(x)=\int_{-\infty}^{\infty}(x-\xi) \exp [-|x-\xi|] f(\xi) d \xi
$$

and we now calculate the derivatives of $D(\cdot)$. First of all,

$$
\begin{aligned}
\frac{d D(x)}{d x} & =\frac{d}{d x}\left\{\int_{-\infty}^{x}(x-\xi) \exp [-(x-\xi)] f(\xi) d \xi\right. \\
& \left.+\int_{x}^{\infty}(x-\xi) \exp [(x-\xi)] f(\xi) d \xi\right\},
\end{aligned}
$$

which reduces to

$$
\begin{aligned}
\frac{d D(x)}{d x} & =\int_{-\infty}^{x}(1-x+\xi) \exp [-(x-\xi)] f(\xi) d \xi \\
& +\int_{x}^{\infty}(1+x-\xi) \exp [(x-\xi)] f(\xi) d \xi .
\end{aligned}
$$


Continuing with higher derivatives we get

$$
\begin{aligned}
\frac{d^{2} D(x)}{d x^{2}}= & \int_{-\infty}^{x}(-2+x-\xi) \exp [-(x-\xi)] f(\xi) d \xi \\
& +\int_{x}^{\infty}(2+x-\xi) \exp [(x-\xi)] f(\xi) d \xi \\
\frac{d^{3} D(x)}{d x^{3}}= & -4 f(x)+\int_{-\infty}^{x}(3-x+\xi) \exp [-(x-\xi)] f(\xi) d \xi \\
& +\int_{x}^{\infty}(3+x-\xi) \exp [(x-\xi)] f(\xi) d \xi, \\
\frac{d^{4} D(x)}{d x^{4}}= & -4 f^{\prime}+\int_{-\infty}^{x}(-4+x-\xi) \exp [-(x-\xi)] f(\xi) d \xi \\
& +\int_{x}^{\infty}(4+x-\xi) \exp [(x-\xi)] f(\xi) d \xi .
\end{aligned}
$$

Combining these expressions into a differential operator

$$
L D=\frac{d^{4} D}{d x^{4}}-2 \frac{d^{2} D}{d x^{2}}+D,
$$

we get the differential equation

$$
L D=-4 f^{\prime}
$$

for the growth power $f$.

Now let us turn to the $2 \mathrm{D}$ case. Then we also need, similar to the above,

$$
\begin{aligned}
h(x)= & \int_{-\infty}^{\infty} \exp [-|x-\xi|] g(\xi) d \xi \\
\frac{d h}{d x}(x)= & -\int_{-\infty}^{x} \exp [-(x-\xi)](g \xi) d \xi \\
& +\int_{x}^{\infty} \exp [(x-\xi)] g(\xi) d \xi \\
\frac{d^{2} h}{d x^{2}}= & -2 g(x)+\int_{-\infty}^{x} \exp [-(x-\xi)] g(\xi) d \xi \\
& +\int_{x}^{\infty} \exp [(x-\xi)] g(\xi) d \xi
\end{aligned}
$$

so that $\frac{d^{2} h}{d x^{2}}-h=-2 g$.

Combining these relations we get the

Proposition. The growth power $f$ in $2 \mathrm{D}$ with $d(\cdot)$ given in terms of the $l_{1}$-norm satisfies the system of partial differential equations

$$
\begin{aligned}
& \frac{\partial^{2}}{\partial x_{2}^{2}}\left\{\frac{\partial^{4} D_{1}}{\partial x_{1}^{4}}-2 \frac{\partial^{2} D_{1}}{\partial x_{1}^{2}}+D_{1}\right\}=8 \frac{\partial f}{\partial x_{2}} \\
& \frac{\partial^{2}}{\partial x_{1}^{2}}\left\{\frac{\partial^{4} D_{2}}{\partial x_{1}^{4}}-2 \frac{\partial^{2} D_{2}}{\partial x_{2}^{2}}+D_{2}\right\}=8 \frac{\partial f}{\partial x_{1}} .
\end{aligned}
$$


WARNING. The appearance of differential operators of high order may cause numerical trouble due to noise in the observations. The equations ought therefore to be modified to a smoothed form by regularization. Also, it may be noticed that the two PDEs are not exactly satisfied, nor exactly equivalent due to biological noise. In particular, we cannot expect agreement between the mixed second derivatives, $\frac{\partial^{2} f}{\partial x_{1} \partial x_{2}}=\frac{\partial^{2} f}{\partial x_{2} \partial x_{1}}$, for the two expressions. So let us write

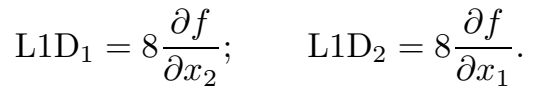

In order to force agreement we look for the $D^{*}=\left(D_{1}^{*}, D_{2}^{*}\right)$ that minimizes the $l_{2}$ norm $\left\|D^{*}-D\right\|$ for $D^{*} \in \mathcal{M}$ in the linear manifold

$$
\mathcal{M}=\left\{D^{*}: \frac{\partial L_{1} D_{1}^{*}}{\partial x_{2}}=\frac{\partial L_{2} D_{2}^{*}}{\partial x_{1}}\right\}
$$

The solution to this minimum problem is of course given by the projection

$$
D^{*}=P_{\mathcal{M}} D,
$$

where the projection operator $P$ is defined in the geometry of the Hilbert space associated with the problem. The numerical version of the problem deals, of course, with a finitedimensional Hilbert space.

The above solution possesses a certain elegance but its applicability is too limited. We shall therefore study a more general approach in the next section.

9.1.2. General estimate of growth power. Still assuming that we have calculated the growth fields $x(\xi, t), \xi \in \Xi, t \in[0, T]$, let us determine the growth amplitude measures $A^{\alpha}(d \eta, t)$ by a more general method. We shall use the general form of the thermodynamic limit for the growth process, allowing it to be time dependent,

$$
\frac{\partial x(\xi, t)}{\partial t}=\sum_{\alpha} \int_{\Xi} \theta^{\alpha}(x(\xi, t)-x(\eta, t)) A^{\alpha}(d \eta, t) .
$$

We believe that sufficient generality will be achieved if we employ just two growth modes, pure growth $\alpha=+$, and pure decay $\alpha=-$, both isotropic so that their respective $k$ values do not depend upon direction; $k=+1$ or -1 . Then growth will be represented by the integro-differential equation

$$
\frac{\partial x(\xi, t)}{\partial t}=\int_{\Xi} \theta^{+}(x-\eta) A^{+}(d \eta, t)+\int_{\Xi} \theta^{-}(x-\eta) A^{-}(d \eta, t) .
$$

Using the form of $\theta(\cdot)$ from Section 5.2.2 this reduces to

$$
\begin{aligned}
\frac{\partial x(\xi, t)}{\partial t}= & \int_{\Xi}(x-\eta) \phi(x-\eta) A^{+}(d \eta, t) \\
& -\int_{\Xi}(x-\eta) \phi(x-\eta) A^{-}(d \eta, t)=\int_{\Xi}(x-\eta) \phi(x-\eta) A^{\text {signed }}(d \eta, t),
\end{aligned}
$$

where we have introduced the signed measure

$$
A^{\text {signed }}(d \eta, t)=A^{+}(d \eta, t)-A^{-}(d \eta, t),
$$


which will actually be handled as if it were absolutely continuous with a density $a^{\text {signed }}(\eta)$; this makes sense computationally. Recall that we have advocated the choice $\phi(y)=$ $\exp \left[-\|y\|^{2} / S T E P\right]$.

In terms of the integral kernel $K(z)=z \phi(z)$, we have the relation

$$
\frac{\partial x(\xi, t)}{\partial t}=\int_{\Xi} K[x(\xi, t)-x(\eta, t)] a^{\text {signed }}(\eta) d \eta .
$$

Treating the growth fields $x(\xi, t)$ as known, perhaps estimated by a technique presented later, we shall solve the equation in (9.21) for the growth amplitude measure by Fourier transforms of the form

$$
\bar{f}(\lambda)=\int_{\Xi} \exp [i\langle\xi, \lambda\rangle] f(\xi) d \xi
$$

for some integrable function $f(\xi), \xi \in \Xi$, and with the wave number $\lambda=\left(\lambda_{1}, \lambda_{2}\right)$. Hence

$$
\frac{\partial}{\partial t} \bar{x}(\lambda, t)=\bar{K}(\lambda, t) \bar{a}(\lambda) ; \quad \bar{a}(\lambda)=\int_{\Xi} \exp (i\langle\xi, \lambda\rangle) a^{\text {signed }}(\xi) d \xi
$$

Note that $\bar{x}, \bar{K}$ are 2-vector-valued while $\bar{a}$ is scalar-valued. We get the two obvious (but perhaps not the best) solutions

$$
a_{1}^{*}(\lambda)=\frac{\frac{\partial}{\partial t} \bar{x}_{1}(\lambda, t)}{\bar{K}_{1}(\lambda)} ; \quad a_{2}^{*}(\lambda)=\frac{\frac{\partial}{\partial t} \bar{x}_{2}(\lambda, t)}{\bar{K}_{2}(\lambda)}
$$

We have for the first component of $\bar{K}$,

$$
\begin{aligned}
\bar{K}_{1}(\lambda) & =\int_{\Xi} \xi_{1} \exp \left(-\|\xi\|^{2} / 2+i\langle\lambda, \xi\rangle\right) d \xi \\
& =-i \frac{\partial}{\partial \lambda_{1}} \int_{\Xi} \exp \left(-\|\xi\|^{2} / 2+i\langle\lambda, \xi\rangle\right) d \xi
\end{aligned}
$$

which reduces to

$$
-i 2 \pi \frac{\partial}{\partial \lambda_{1}} \exp \left(-\|\lambda\|^{2} / 2\right)=-i 2 \pi \lambda_{1} \exp \left(-\|\lambda\|^{2} / 2\right) .
$$

Combining the two solutions gives

$$
a^{*}(\lambda)=\frac{a_{1}^{*}(\lambda)+a_{2}^{*}(\lambda)}{2}
$$

Theoretically this is acceptable, but computationally the resulting algorithm must be modified; see Grenander (1981), Chapter 7. Indeed, we ought to regularize the estimate by multiplying it by a function $R(\lambda)$ before applying Fourier inversion. The function $R$ should be close to one in a big neighborhood of zero in $\mathbf{R}^{2}$ and then tend to zero for very large values of $\lambda$.

REMARK. For concreteness we have used Fourier integrals, but in practice the set $\Xi$ will be compact, so that it will be more natural to use Fourier series. 
9.2. Inference for biological mappings. To prepare for image inference based on the GRID model let us consider the posterior energy expressed in terms of the cell activities as represented by $F^{*}$ :

$$
E_{\text {posterior }}\left(F^{*}\right)=-\log L\left(F^{*}\right)-\log p_{\text {prior }}\left(F^{*}\right) .
$$

With standard assumptions we have

$$
-\log L\left(F^{*}\right)=\frac{1}{2 \sigma^{2}}\left\|I^{D}-x^{-1} \circ I_{\text {temp }}\right\|^{2}
$$

and, appealing to the properties of the Poisson process,

$$
\begin{aligned}
-\log p_{\text {prior }}\left(F^{*}\right) & =-\log \left\{\prod _ { i = 1 } ^ { n } \Lambda \left(\eta_{i} \times \exp \left[-\int_{\eta \in \Xi} \Lambda\left(\eta_{i}\right) d \eta\right\}\right.\right. \\
& \left.=-\sum_{i=1}^{n} \log \Lambda\left(\eta_{i}\right)+\int_{\eta \in \Xi} \Lambda(\eta) d \eta\right\} .
\end{aligned}
$$

The last expression becomes

$$
-\log p_{\text {prior }}\left(F^{*}\right)=\int_{\eta \in \Xi}\left[-F^{*}(d \eta)+\Lambda(\eta) d \eta\right] .
$$

This differs from the form of the posterior energy used earlier in that the term (9.29) replaces that of a Sobolev integral; for example, in (2.1), equation (9.21) is biologically motivated in contrast to the Sobolev expression that is selected more for its mathematical convenience than for its realism.

Now let us examine a measure of growth tendency via a particular variation in the diffeomorphism. Consider the expression

$$
E=\int_{X}\left[I_{\text {new }}(x)-\left(\phi \circ I_{\text {old }}\right)(x)\right]^{2} d x,
$$

where $I_{o l d}=\phi \circ I_{\text {old }}$ is the result of the growth process during the time interval represented by a diffeomorphism $\phi$, and $I_{n e w}$ is the organism at the beginning of a (short) time period $\left(t_{1}, t_{2}\right)$ of growth. Writing $\phi=i d+\epsilon \psi$ we get the asymptotics for the change in $E$ over $\left(t_{1}, t_{2}\right)$,

$$
\Delta E=-2 \epsilon \int_{X}\left(\left[I_{\text {new }}-I_{o l d}\right] \circ \psi\right)(x) d x+O\left(\epsilon^{2}\right),
$$

which makes it natural to use the criterion

$$
\text { tendency }=\epsilon \int_{X}\left(\left[I_{\text {new }}-I_{\text {old }}\right] \circ \psi\right)(x) d x
$$

as a measure of the tendency for the organism to grow by the mapping $\psi$.

Note, however, that (real) growth along a curve $\Gamma \subset X$ can also be obtained by decay along another curve $\Gamma^{\text {prime }}$ more or less parallel to $\Gamma$. This growth duality makes it clear that the explanation of an observed growth will not be unique.

It will be instructive to look at a real case. In Figure 15 we show $I_{\text {old }}$ and $I_{n e w}$ in the upper two panels. The lower left panel shows the growth tendency computed for the two given images, and one can see clearly the effect of the dualism. The lower right panel indicates real growth. 
The family $\Phi$ of diffeomorphisms is algebraically closed under composition, but is that the case with addition of the vector fields associated with the different diffeomorphisms? Say that we are interested in growth along a $C_{2}$-curve $\Gamma$ that carries a continuum of seeds, for example starting from the maps $x \rightarrow x^{\prime}=\xi+\psi(\|x-\xi\|)$. Consider addition over the continuum $\Gamma$,

$$
x \rightarrow x^{\prime}=\int_{\Gamma}[\xi(s)+\psi(\|x-\xi(s)\|)[x-\xi(s)]] \gamma(s) d s
$$

with $d s$ meaning arc length and $\gamma(\cdot)$ being a density with respect to arc length. Now, consider another point $y \neq x$ and its map via addition of the same vector field

$$
y \rightarrow y^{\prime}=\int_{\Gamma}[\xi(s)+\psi(\|y-\xi(s)\|)[y-\xi(s)]] \gamma(s) d s .
$$

Can it happen that $x$ and $y$ are mapped into the same point? If they are, we have

$$
\int_{\Gamma} \psi(\|x-\xi(s)\|)[x-\xi(s)] \gamma(s) d s=\int_{\Gamma} \psi(\|y-\xi(s)\|)[y-\xi(s)] \gamma(s) d s .
$$

We do not have the general answer to this question, but for the isotropic map in Section 5.2 with $\psi \equiv k,(9.37)$ reduces to

$$
x k \int_{\Gamma} \gamma(s) d s-\int_{\Gamma} \xi(s) \gamma(s) d s=y k \int_{\Gamma} \gamma(s) d s-\int_{\Gamma} \xi(s) \gamma(s) d s
$$

implying that $x=y$, a contradiction, so that we have a bijective map.

Lemma. The mappings $x \rightarrow \xi+k(x-\xi)$ combined over different seeds via addition yield diffeomorphic mappings.

9.3. Estimation using group cascades. Let us now begin a more systematic study of how to estimate the mapping in a GRID model calling on the doctrine of group cascades; see Matejic (1997). We shall employ the following cascade:

1) a biological transformation with an approximation for short duration $t_{2}-t_{1}$ for the thermodynamic limit:

$$
\text { GRID }: x\left(\xi, t_{2}\right) \approx x\left(\xi, t_{1}\right)+\int_{\eta \in \Xi}\left[x\left(\xi, t_{1}\right)-x\left(\eta, t_{1}\right)\right] \phi\left(\left\|x\left(\xi, t_{1}\right)-x\left(\eta, t_{1}\right)\right\|\right) a(\eta) d \eta
$$

2) a registration transformation $E G(2): x \mapsto x+c+O x$; a 2 -vector $c$ and a $2 \times 2$ orthogonal matrix $O$, with some convention for dealing with $x$-values outside $X$;

3) a photometric transformation $\mathbf{R}_{+}: I(x) \mapsto k I(x)$. This is probably too limited; a more flexible group of photometric transformations is needed;

4) observational noise $\mathbf{R}(l \times l): I(x) \mapsto I(x)+i . i . d$. noise $N\left(0, \sigma_{\text {obs }}^{2}\right)$.

With the two observed images $I_{1}, I_{2}$ at time points $t_{1}, t_{2}$, respectively, we shall minimize the total energy $E_{\text {total }}(a)$ as a function of the energy parameters; the energy space $\mathcal{E}$ is $\left\{a(\cdot) \in \mathbf{R}^{l 1 \times l 2}\right\} \times\left\{c \in \mathbf{R}^{2}\right\} \times\{v \in \mathbf{R}\} \times\left\{k \in \mathbf{R}_{+}\right\}$. The energy is made up of contributions from each of the groups in the cascade:

$$
E_{\text {total }}(a)=E_{\text {biol }}(a)+E_{\text {reg }}(c, O)+E_{\text {photo }}(c)+E_{\text {obs }}(a)
$$

with the prior energies $E_{b i o l}, E_{r e g}, E_{\text {photo }}(c)$ and the likelihood energy $E_{o b s}$. 
We shall assume that the biological energy has $a$-components $=i . i . d . \quad N\left(0, \sigma_{\text {biol }}\right)$ so that

$$
E_{\text {biol }}(a)=\frac{1}{2 \sigma_{\text {biol }}^{2}} \int_{\eta \in \Xi} a^{2}(\eta) d \eta,
$$

which is really too arbitrary and should be replaced by a more realistic assumption when we have learned more about the applications of GRID.

Furthermore, the registration energy with $c=N\left(0, \sigma_{c}^{2}\right), v=N\left(0, \sigma_{v}^{2}\right)$, and $O=$ $\left(\begin{array}{cc}\cos v & \sin v \\ -\cos v & \cos v\end{array}\right)$ is such that

$$
E_{r e g}(c, O)=\frac{c^{2}}{2 \sigma_{c}^{2}}+\frac{v^{2}}{2 \sigma_{v}^{2}}
$$

and

$$
E_{\text {photo }}(k)=\frac{k^{2}}{2 \sigma_{\text {photo }}^{2}} .
$$

Finally, the observation noise energy is

$$
E_{o b s}(a)=\frac{1}{2 \sigma_{\text {obs }}^{2}} \int_{\Xi}\left\{k I_{1}\left[x\left(\xi, t_{1}\right)+c+O x\left(\xi, t_{1}\right)-I_{2}\left[x\left(\xi, t_{2}\right)\right]\right\}^{2} \mu(d \xi) .\right.
$$

We shall base the estimation of diffeomorphism on a gradient algorithm, either deterministic or of Langevin form for stochastic relaxation. In both cases we need the energy gradients. The prior energies are easily dealt with: the biological

$$
\frac{\partial E_{b i o l}}{\partial a(\eta)}=\frac{a(\eta)}{\sigma_{b i o l}^{2}}
$$

and the registration

$$
\frac{E_{r e g}}{\partial c}=\frac{c}{\sigma_{c}^{2}}, \quad \frac{\partial E_{r e g}}{\partial v}=\frac{v}{\sigma_{v}^{2}}
$$

as well as the photometric

$$
\frac{\partial E_{\text {photo }}}{\partial k}=\frac{k}{\sigma_{\text {photo }}^{2}} .
$$

The gradient of the likelihood energy is a little tricky:

$$
\begin{aligned}
\frac{\partial E_{o b s}}{\partial a(\eta)} & =\frac{1}{\sigma_{o b s}^{2}} \int_{\Xi}\left\{k I_{1}\left[x\left(\xi, t_{1}\right)+c+O x\left(\xi, t_{1}\right)-I_{2}\left[x\left(\xi, t_{2}\right)\right]\right\}\right. \\
& \times k \frac{\partial I_{1}\left[x\left(\xi, t_{1}\right)+c+O x\left(\xi, t_{1}\right)-I_{2}\left[x\left(\xi, t_{2}\right)\right]\right.}{\partial a(\eta)} \mu(d \xi)
\end{aligned}
$$

with the inner derivative

$$
\begin{aligned}
& \frac{\partial I_{1}\left[x\left(\xi, t_{1}\right)+c+O x\left(\xi, t_{1}\right)-I_{2}\left[x\left(\xi, t_{1}\right)\right]\right]}{\partial a(\eta)} \\
& \quad=-\left\langle\operatorname{grad} I_{2}\left(x\left(\xi, t_{2}\right),\left[x\left(\xi, t_{1}\right)-x\left(\eta, t_{1}\right)\right]\right\rangle \phi\left(\left\|x\left(\xi, t_{1}\right)-x\left(\eta, t_{1}\right)\right\|\right)\right),
\end{aligned}
$$

where $\langle$.$\rangle and grad mean the inner product and gradient, respectively, in \mathbf{R}^{2}$. 
Also,

$$
\begin{aligned}
\frac{\partial E_{o b s}}{\partial c_{i}} & =k \frac{1}{\sigma_{o b s}^{2}} \int_{\Xi}\left\{k I _ { 1 } \left[x\left(\xi, t_{1}\right)+c+O x\left(\xi, t_{1}\right)\right.\right. \\
& \left.\left.-I_{2}\left[x\left(\xi, t_{1}\right)\right]\right]\right\} \operatorname{grad}_{i}\left\{I_{1}\left[x\left(\xi, t_{1}\right)+c+O x\left(\xi, t_{1}\right)-I_{2}\left[x\left(\xi, t_{1}\right)\right]\right]\right\} \mu(d \xi) ; i=1,2
\end{aligned}
$$

and

$$
\begin{aligned}
\frac{\partial E_{o b s}}{\partial v}= & k \frac{1}{\sigma_{o b s}^{2}} \int_{\Xi}\left\{k I_{1}\left[x\left(\xi, t_{1}\right)+c+O x\left(\xi, t_{1}\right)-I_{2}\left[x\left(\xi, t_{1}\right)\right]\right]\right\} \\
& \times\left\langle\operatorname{grad} I_{1}\left(\xi, t_{1}\right), O_{1} x\right\rangle \mu(d \xi)
\end{aligned}
$$

with the matrix $O_{1}=\left(\begin{array}{cc}-\sin v & \cos v \\ -\cos v & -\sin v\end{array}\right)$ and finally

$$
\frac{\partial E_{o b s}}{\partial k}=\frac{1}{\sigma_{o b s}^{2}} \int_{\Xi}\left\{k I_{1}\left[x\left(\xi, t_{1}\right)+c+O x\left(\xi, t_{1}\right)-I_{2}\left[x\left(\xi, t_{1}\right)\right]\right]\right\} I_{1}\left(\xi, t_{1}\right) \mu(d \xi) .
$$

The other partial derivatives are zero.

REMARK. If we want the determination of the diffeomorphism to emphasize the near coincidence of the boundaries in $I_{1}$ and $I_{2}$, then we could use a Sobolev norm; for example, adding to (9.44) a term

$$
\begin{gathered}
\frac{1}{2 \sigma_{\text {boundary }}^{2}} \int_{\Xi} \| k\left(\operatorname{grad} I_{1}\right)\left(x\left(\xi, t_{1}\right)+c+O x\left(\xi, t_{1}\right)\right) \\
-\left(\operatorname{grad} I_{2}\right)\left(x\left(\xi, t_{1}\right)\right) \|^{2} \mu(d \xi) .
\end{gathered}
$$

We are dealing with $l \times l+4$ degrees of freedom in the parameter space $\mathbf{P}$. A simple Langevin version of stochastic relaxation takes the form of the stochastic differential equation in $\mathbf{P}$ with the gradient $\operatorname{grad}_{\mathbf{P}}$, i.e.,

$$
p(d \tau)=-\operatorname{grad}_{\mathbf{P}} d \tau+\sqrt{2} W_{l^{2}+4}(d \tau)
$$

with Wiener noise in $\mathbf{P}$. In (9.57) $\tau$ means algorithmic time in contrast to the biological time $t$.

The most severe limitations of this estimation algorithm are the simple-minded assumption 3) about the photometric effect and the use of the short duration approximation to the thermodynamic limit. In 3) we assumed that the different $a(\eta)$ 's were stochastically independent in its prior measure, which will allow the $a(\cdot)$ function to oscillate wildly. It is not clear if this should be accepted, but in a sub-organism the values of the growth power may be quite different from that at neighboring sites, so there is some motivation to allow at least isolated discontinuities. Obviously, this needs more thought. Concerning the photometric effect one ought to take into account the effect of myelination, which can make a difference at least for longer durations.

We have implemented this inference algorithm using only the biological energy term. To translate the continuous version with integrals and derivatives we have applied a 
modicum of numerical analysis to develop some rather primitive software. To test the algorithm we have applied it to a phantom; see Figure 16. It shows the initial $\Omega(0)$ and the grown $\Omega(t)$ together with the Darcyans for both stages. The inference looks excellent, at least qualitatively. A real image is shown in Figure 17, where the estimated growth power is weaker but the growth is still noticeable in the right panels.

An important byproduct of this algorithm is the estimated growth power $a(\cdot)$. We show it in Figure 18 as expressed in $x$-coordinates. Note the color gradient in the figure. We should learn to read such diagrams to get a biologically motivated interpretation.

9.4. Finding anatomical components. Let us assume that we have access to an anatomical doctrine that makes it possible to determine a particular anatomical component $\omega(t)$ in an image $I(t)$. Let us call the doctrine anatom. Suppose we are given an organism $\Omega\left(t_{1}\right)$, imaged as $I 1$, and a later, grown version $\Omega\left(t_{2}\right)$, imaged as $I 2$. If $\phi\left(t_{1}, t_{2}\right)=x\left(\xi, t_{1}\right) \rightarrow x\left(\xi, t_{2}\right), \xi \in \Xi$, is the growth transformation $\phi\left(t_{1}, t_{2}\right): I 1 \rightarrow I 2$, how can we use the knowledge of $\omega\left(t_{1}\right)$ to find $\omega\left(t_{2}\right)$ ? Well, $\omega\left(t_{2}\right)=\phi\left(t_{1}, t_{2}\right) \omega\left(t_{1}\right)$, so that we first find the set $\Xi_{\omega}$ in the Darcyan that represents the component: this set is independent of $t$. In other words, $\omega\left(t_{1}\right)=\left\{x\left(\xi, t_{1}\right) \quad \forall \xi \in \Xi\right\}$. Then we have simply $\omega\left(t_{2}\right)=\left\{x\left(\xi, t_{2}\right) \quad \forall \xi \in \Xi\right\}$.

Applying this procedure and the inference algorithm in Section 8.3 to the two growth images in Figures 19 a) and b), where the area around the anterior commissure in a) is indicated in red, we see the estimated anterior commissure in $\Omega\left(t_{2}\right)$ obtained in b). Careful inspection of the figure shows that the estimated set is indeed the result of the flow of growth through the duration $\left(t_{1}, t_{2}\right)$.

The basis for the above can be expressed by saying that the diagram in Figure 20 commutes, a statement that is not quite as obvious as it may seem at first glance.

9.5. FANOVA for GRID. So far the inferences have been of the estimation type, but there is also a need for tests of significance, for example in experiments with genetic modification and the study of the resulting changes in growth. This could take the form of ANOVA except that in such experiments the parameter space will be of extremely high dimension, in 3D easily of size $2^{20}-2^{30}$, so that we are dealing with essentially infinitedimensional function spaces. This leads to Functional ANOVA, designated FANOVA, a field that has recently attracted a good deal of attention; see, e.g., Antoniadis (2003).

One way of handling the problems associated with the high or infinite dimension is to project the image space, say of GRID type, into a low-dimensional subspace. Using functions from the space $\mathcal{F}$ that we introduced during the construction of Darcyans and the estimated growth power $a(\xi)$ we form an $n$-vector $a=\left(\left\langle a, f_{1}\right\rangle,\left\langle a, f_{2}\right\rangle,\left\langle a, f_{3}\right\rangle\right.$, $\left.\ldots,\left\langle a, f_{n}\right\rangle\right)$, where $\langle\cdot\rangle$ stands for an inner product in the Hilbert space $\mathcal{F}$ containing the growth power functions. Calculating the $a$-vector for each observation we can now carry out ordinary ANOVA in terms of the $a$-data..

As an example we consider a factorial experimental design with animals from different genetic strains, say natural and knock-out mice, with replication and over different time intervals. Graphically we can represent such a design as in Figure21, and we can form the usual quadratic forms from the $a$-vectors. It should be remembered, however, that since the components of the growth power vector are stochastically dependent, the significance levels attained should be interpreted with some caution. 


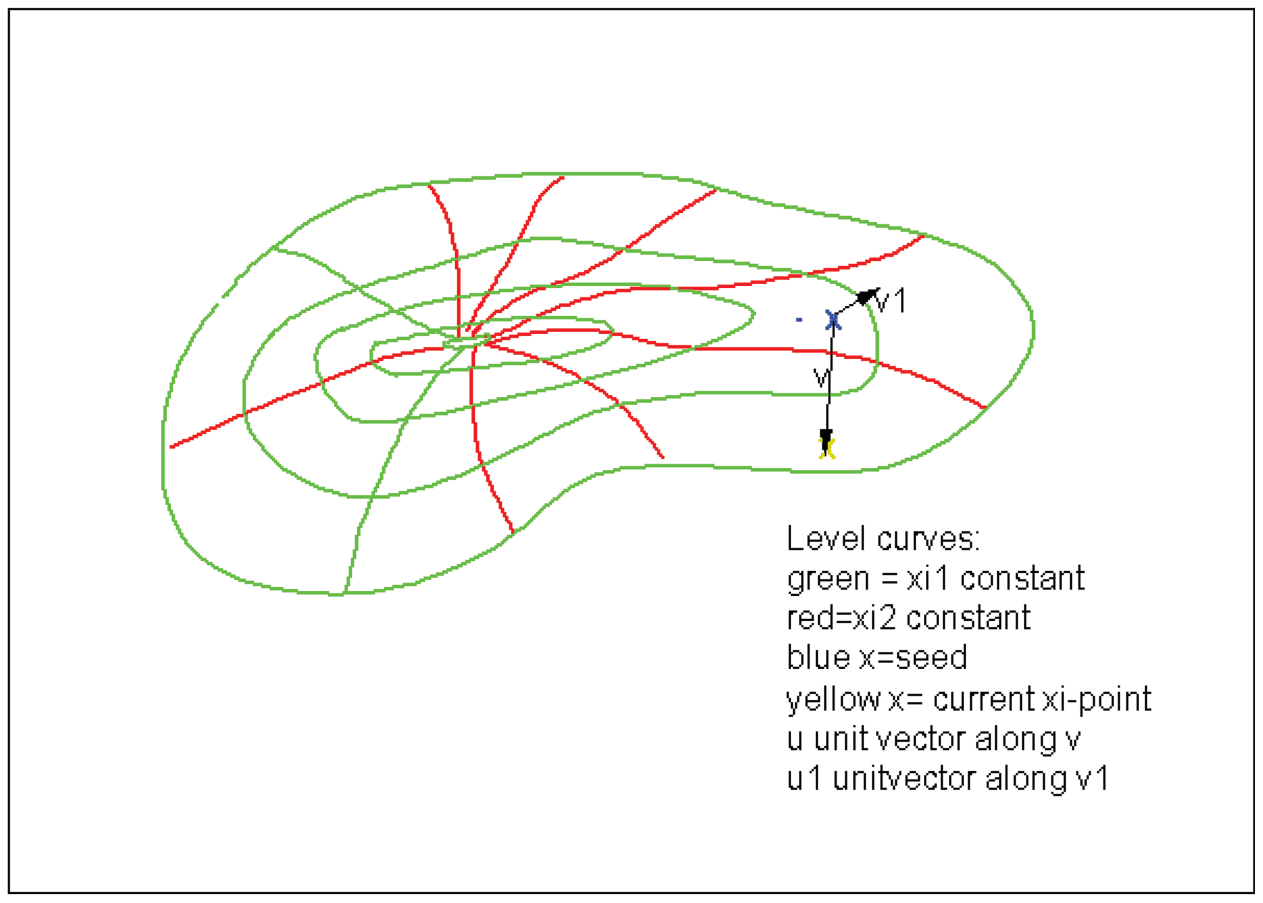

FIG. 1. 


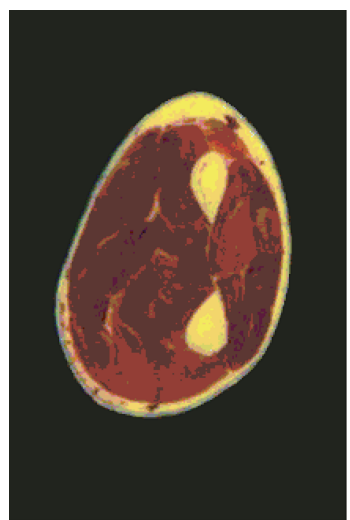

(a)

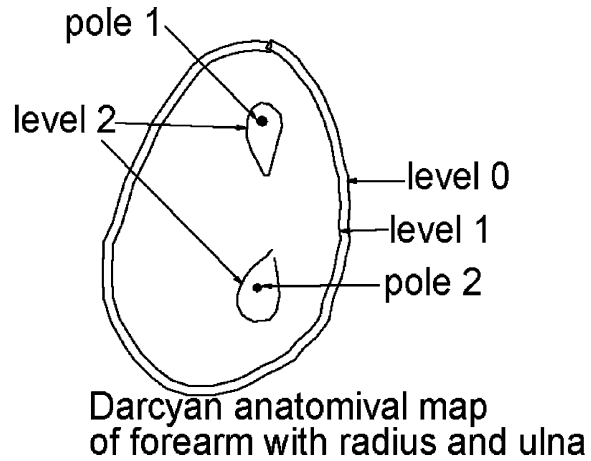

(b)

FIG. 2.

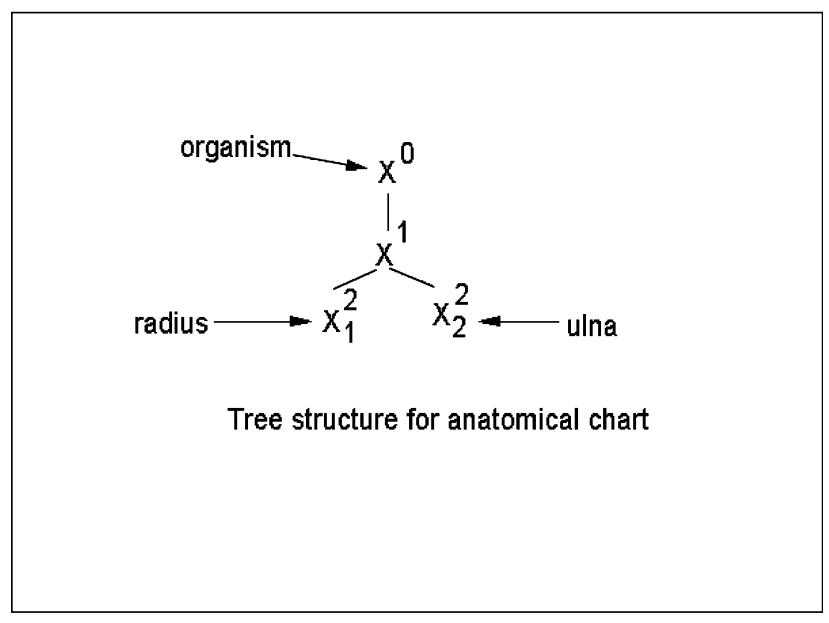

FIG. 3. 

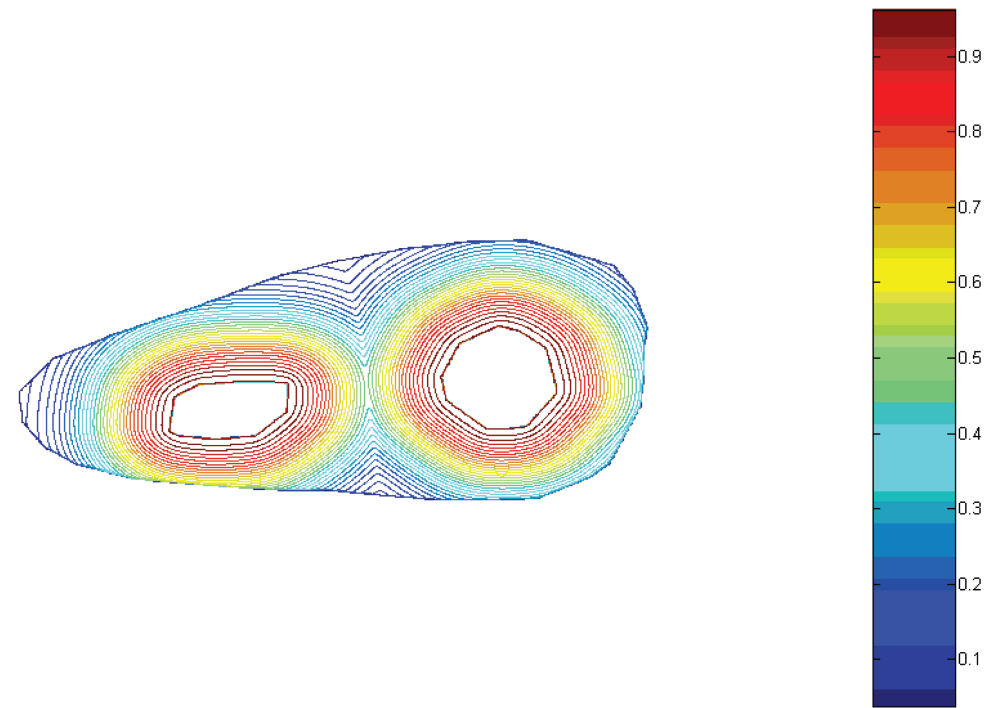

FIG. 4. 


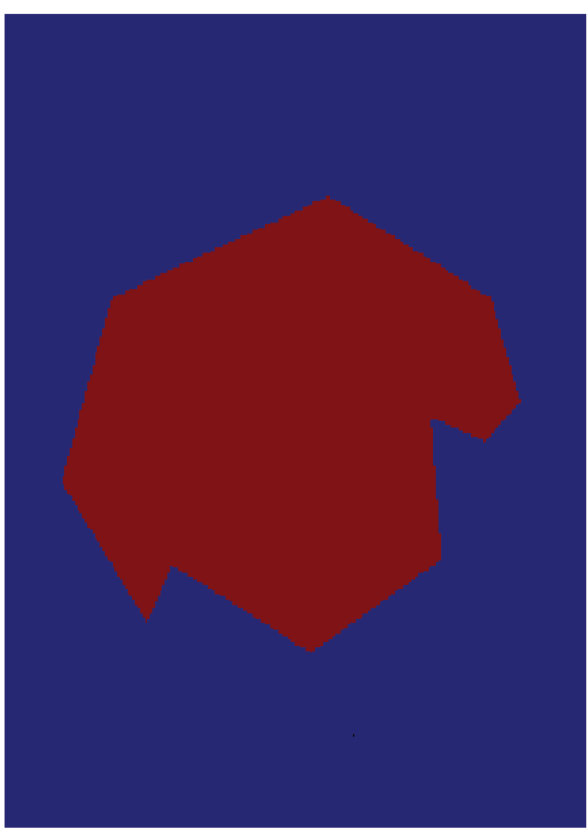

(a)

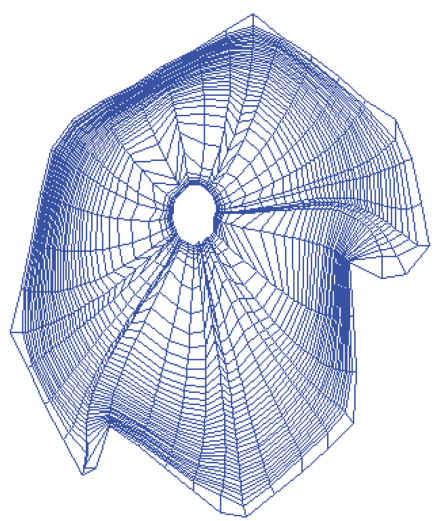

(b)

FIG. 5.
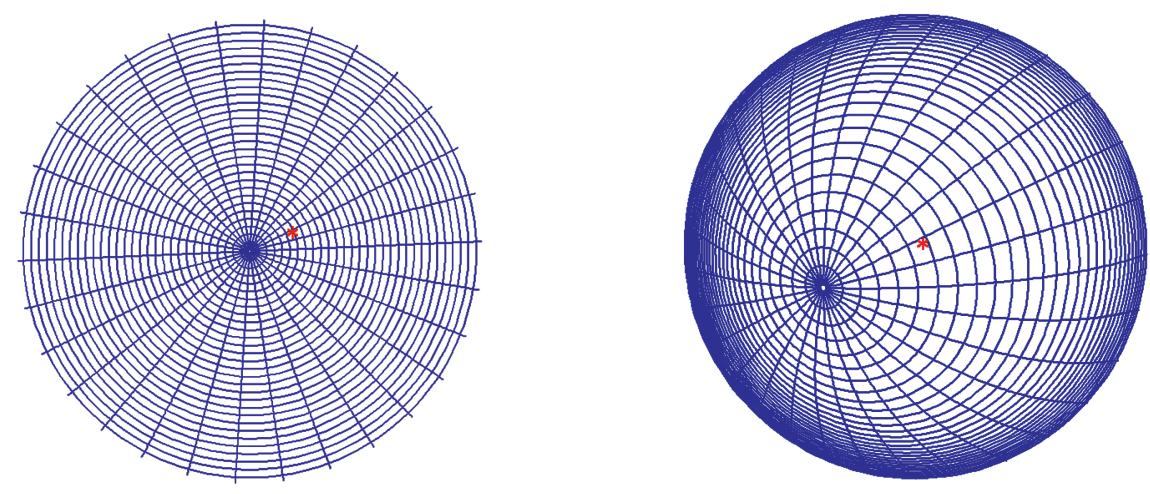

FIG. 6 . 

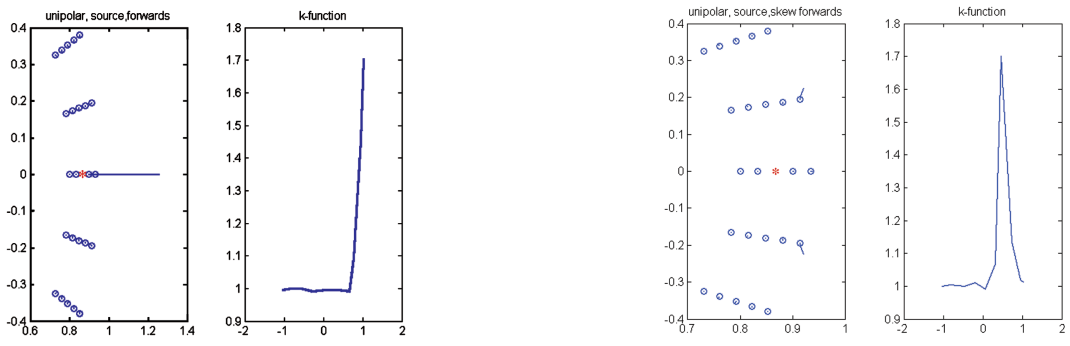

FIG. 7. a. Left panels: one source, deformation forward (all directions with respect to $\xi_{1}$-axis). Right panels: one source, deformation skew forward.
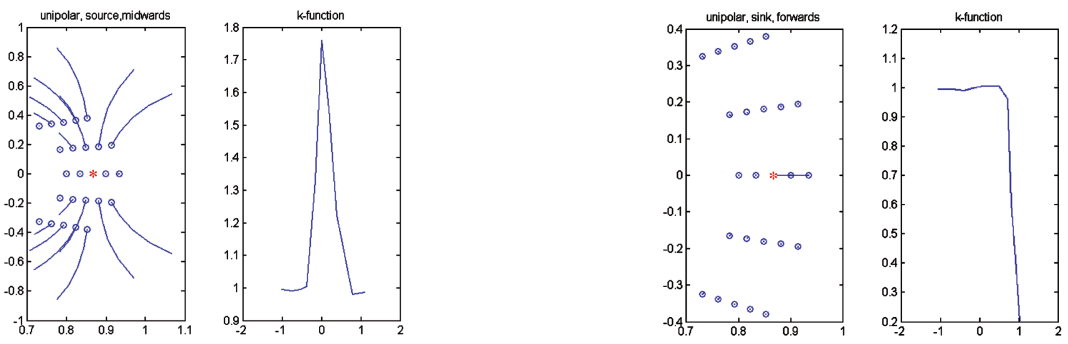

FIG. 7. b. Left panels: one source deformation midward. Right panel: one sink deformation forward.
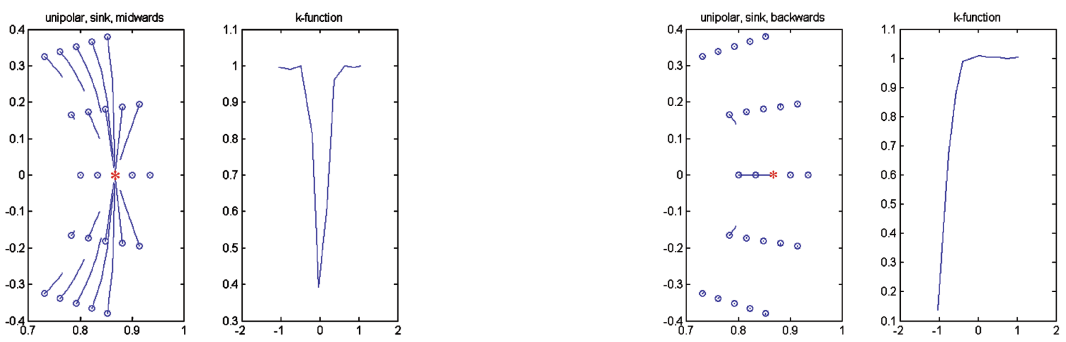

FIG. 7. c. Left panels: one sink, deformations midward. Right panels: one sink, deformation backwards. 

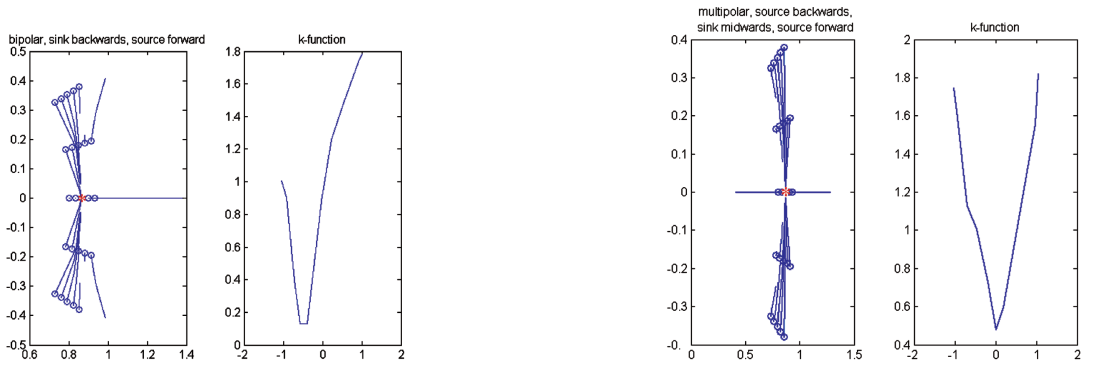

FIG. 7. d. Left panels: one sink, deformation backwards, one source deformations forward. Right panels: one source backwards, one sink midwards, one source forward.
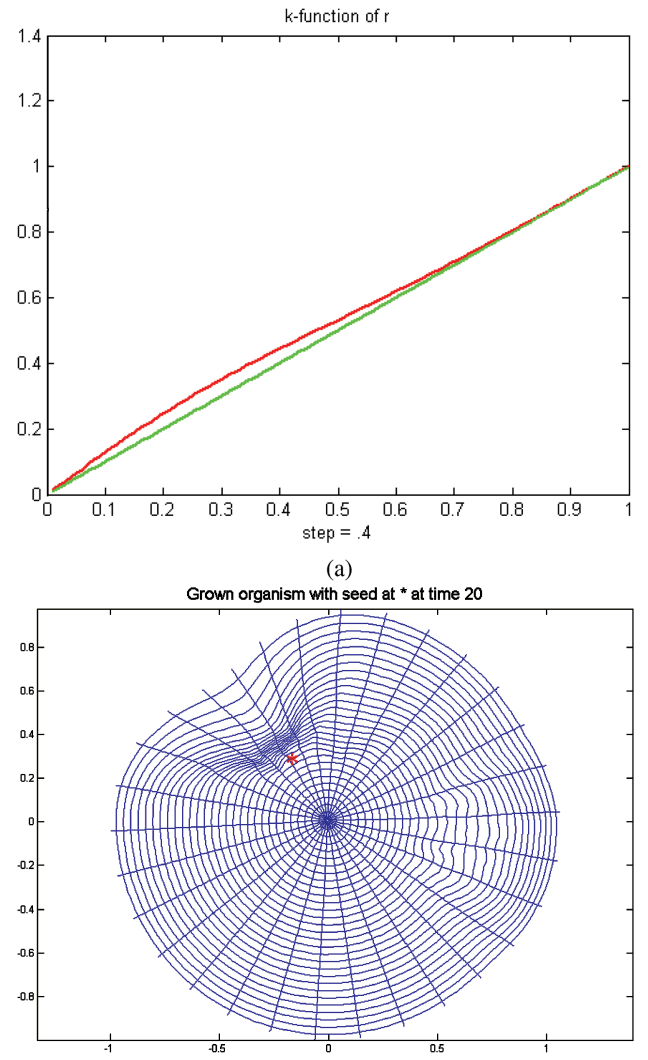

FIG. 8. 


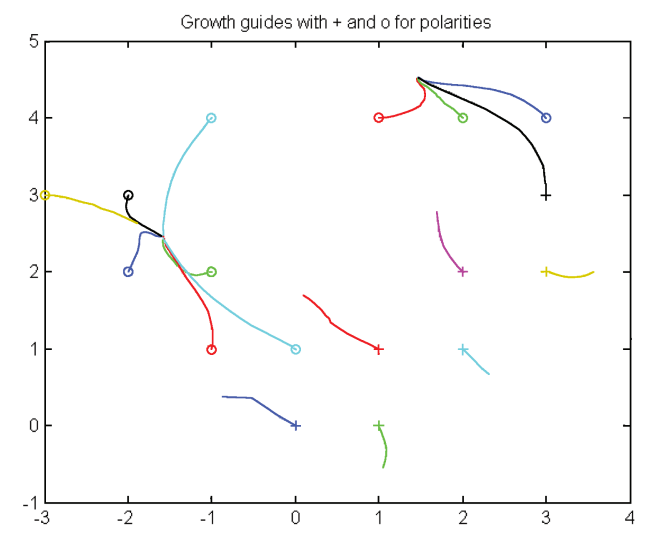

Fig. 9.
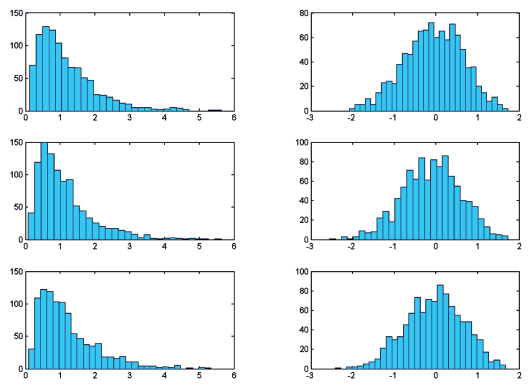

FIG. 10
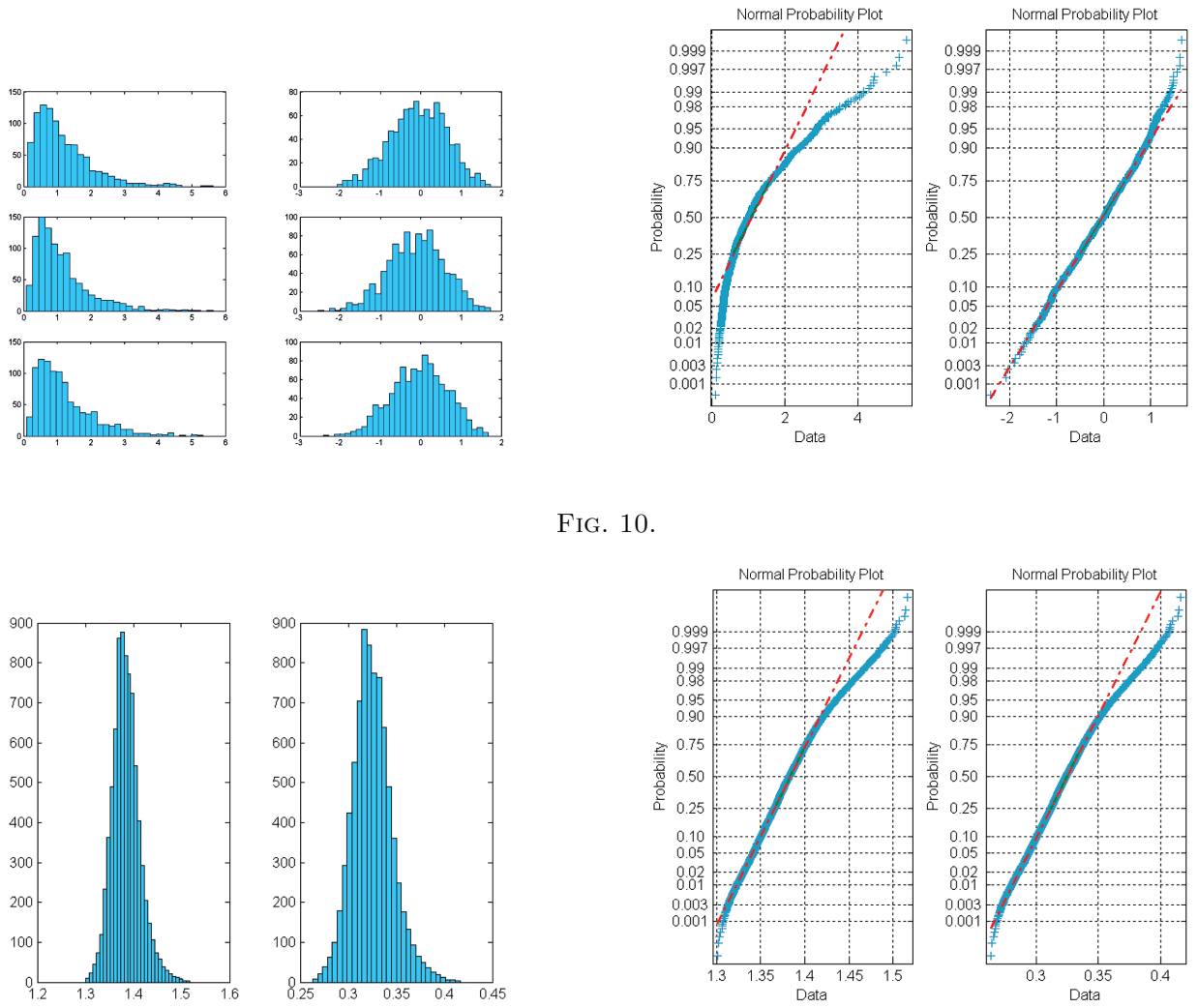

FIG. 11. 

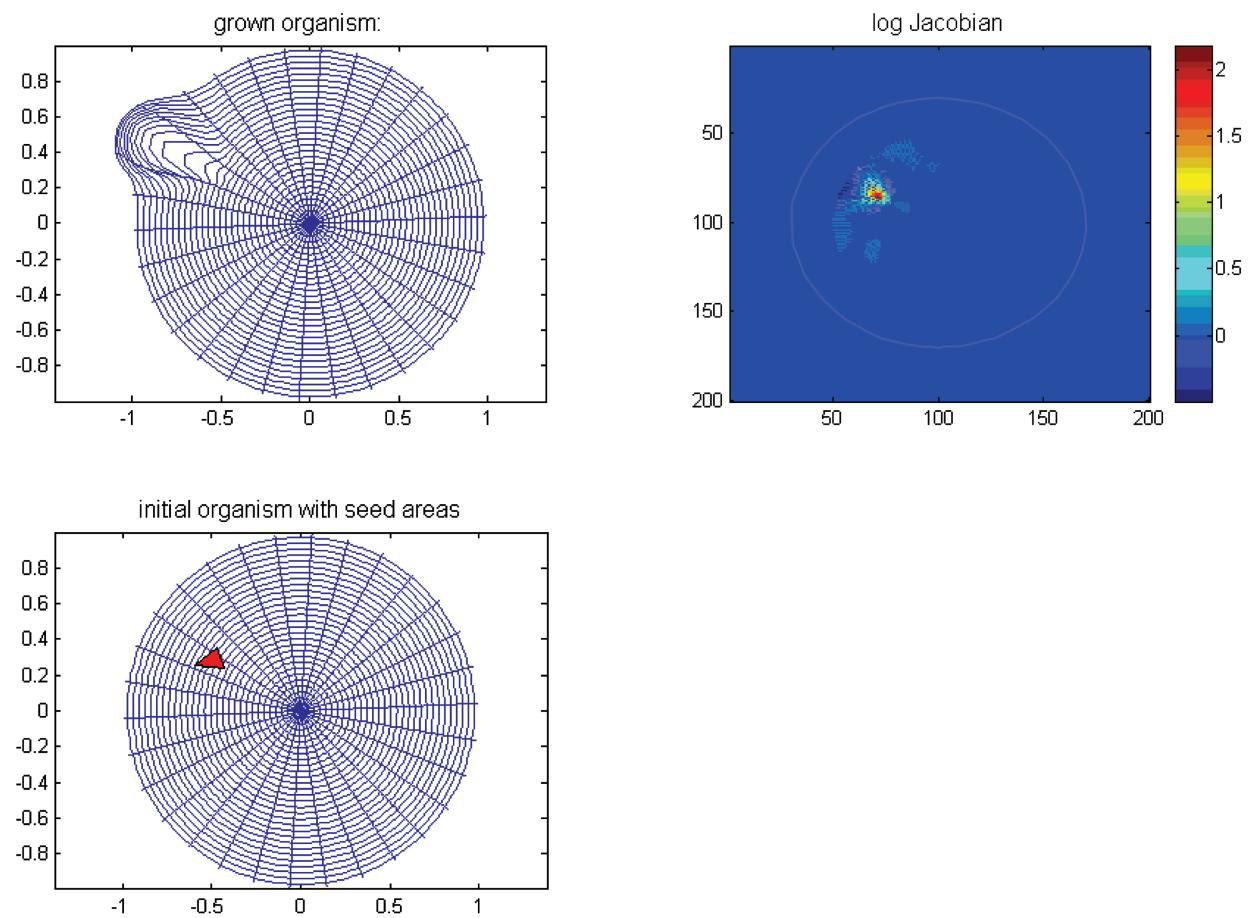

FIG. 12. 

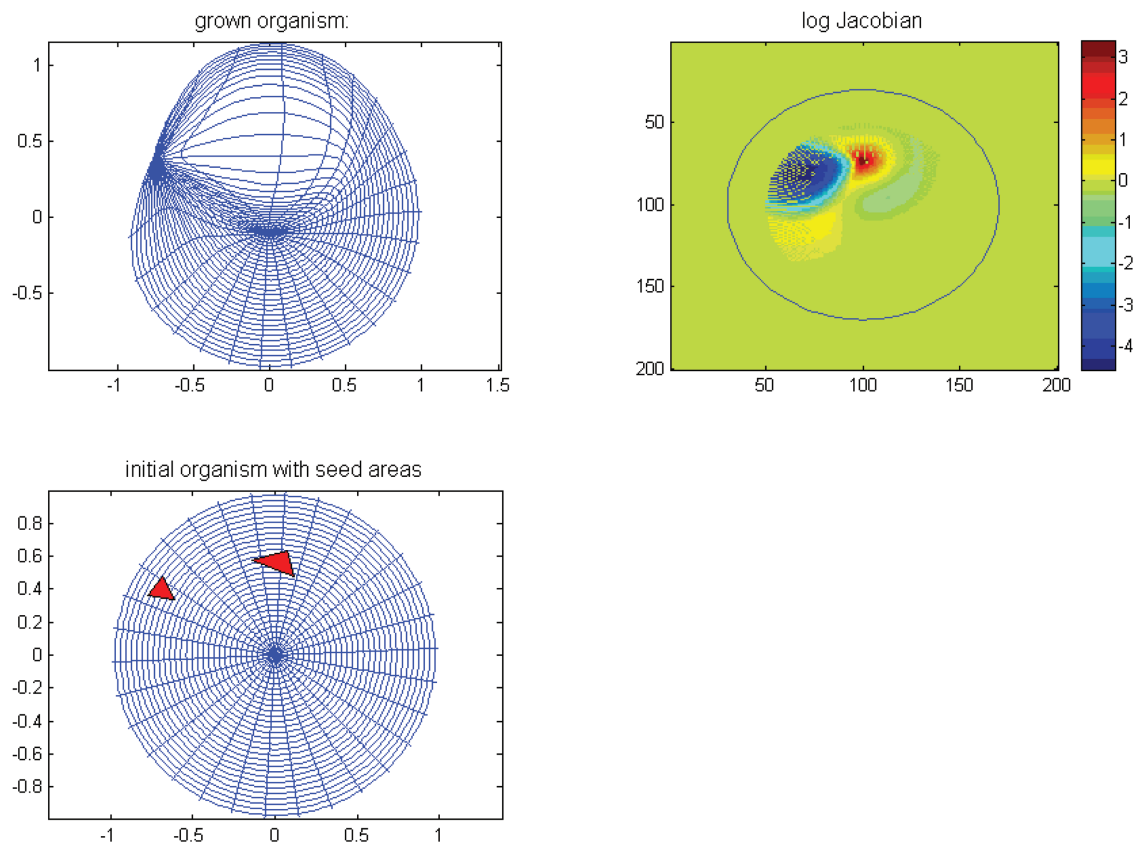

FIG. 13. 

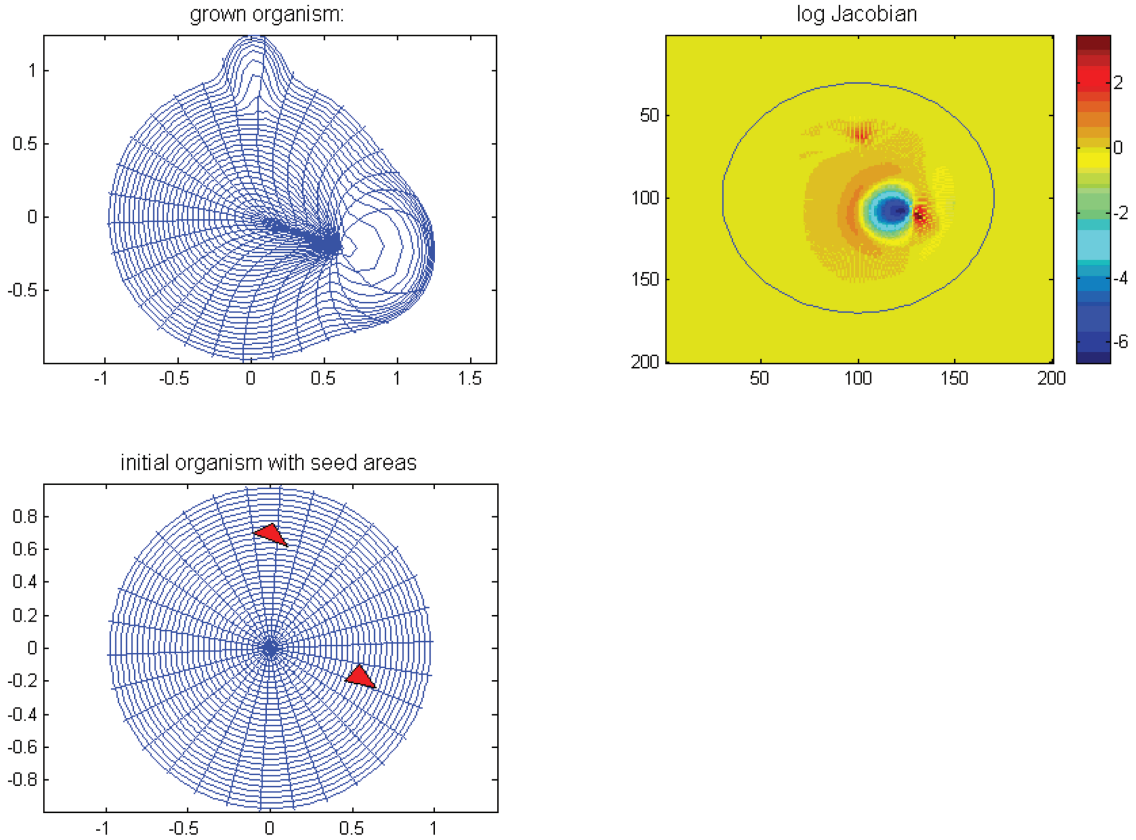

FIG. 14. 

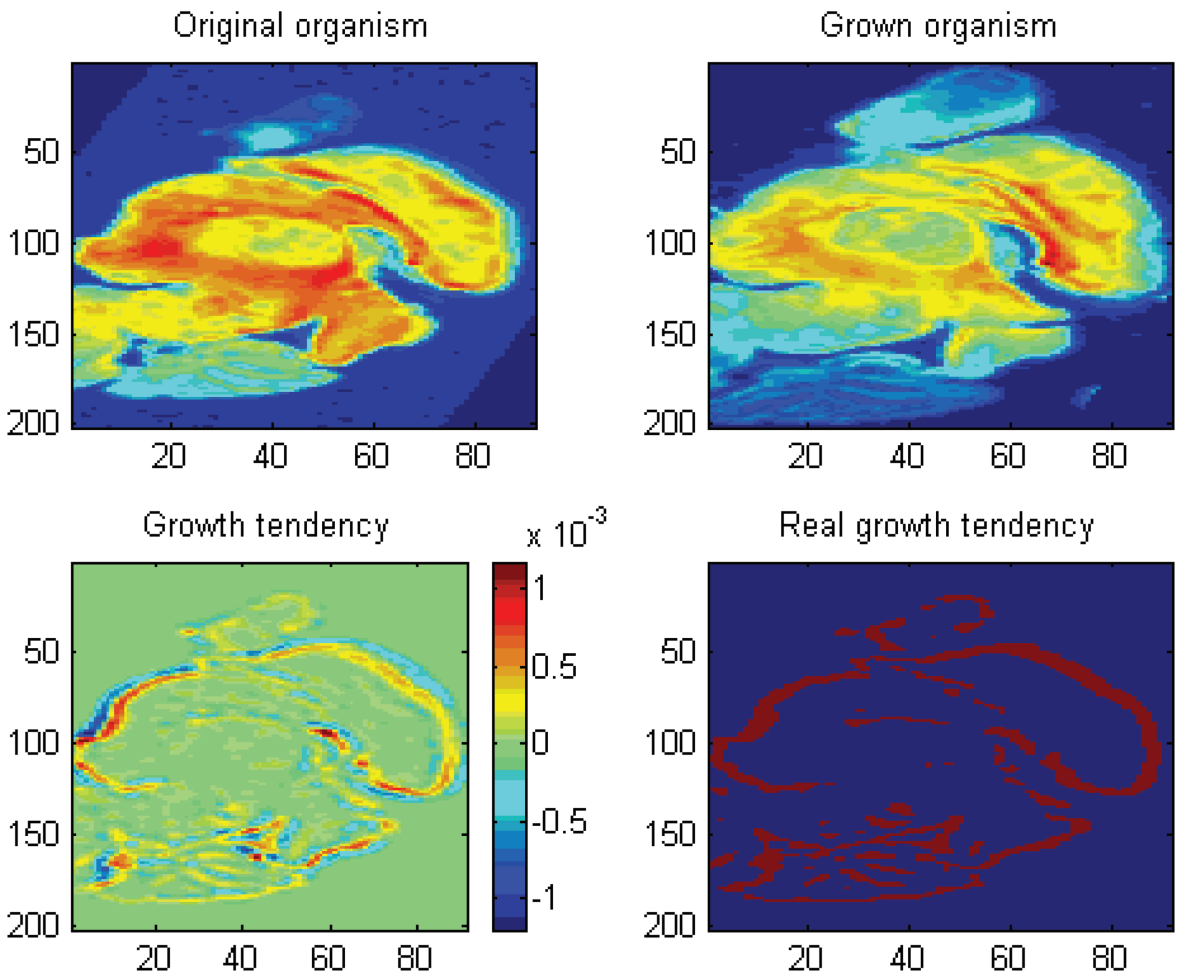

FIG. 15

(a): old orgamism I1
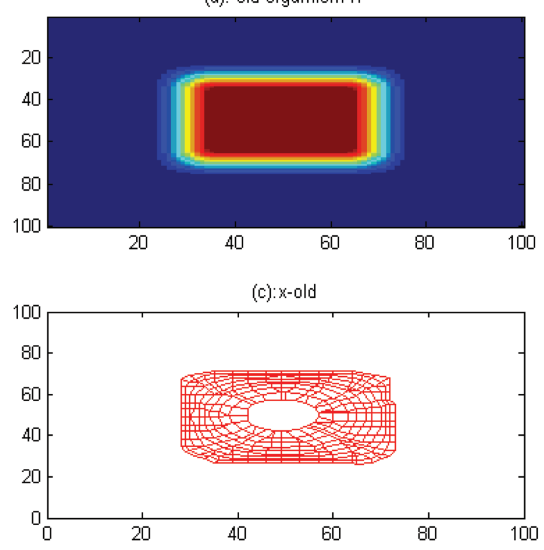

(b): new organism 12
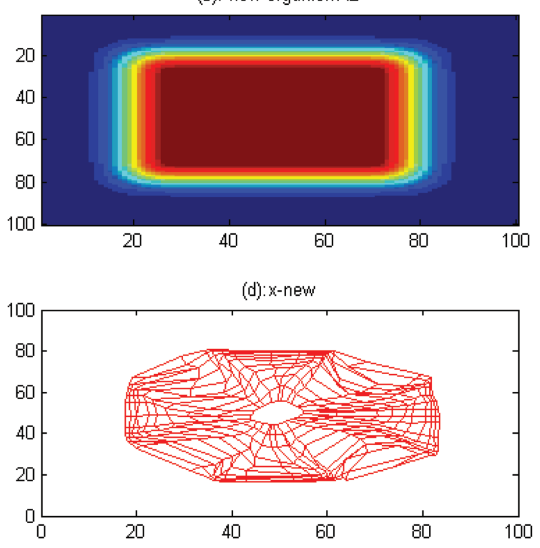

FIG. 16. 
(a): old orgamism I1
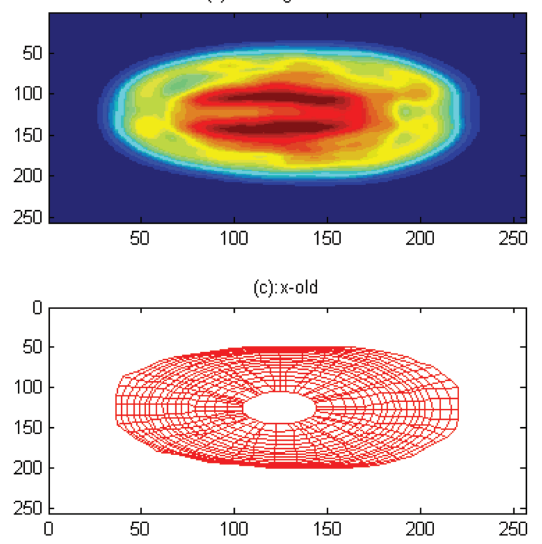

(b): new organism 12

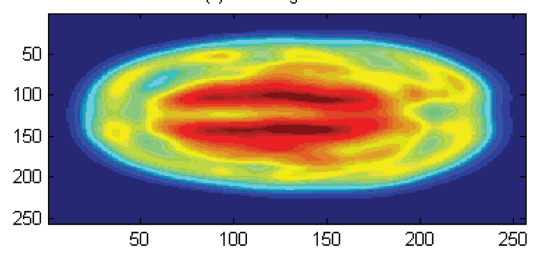

(d): $x$-new

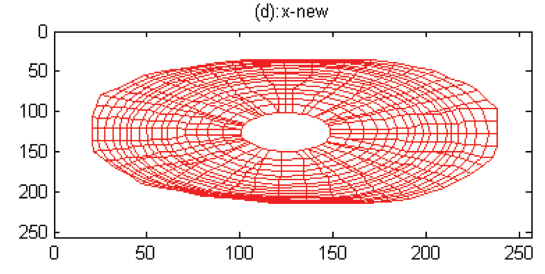

FIG. 17.

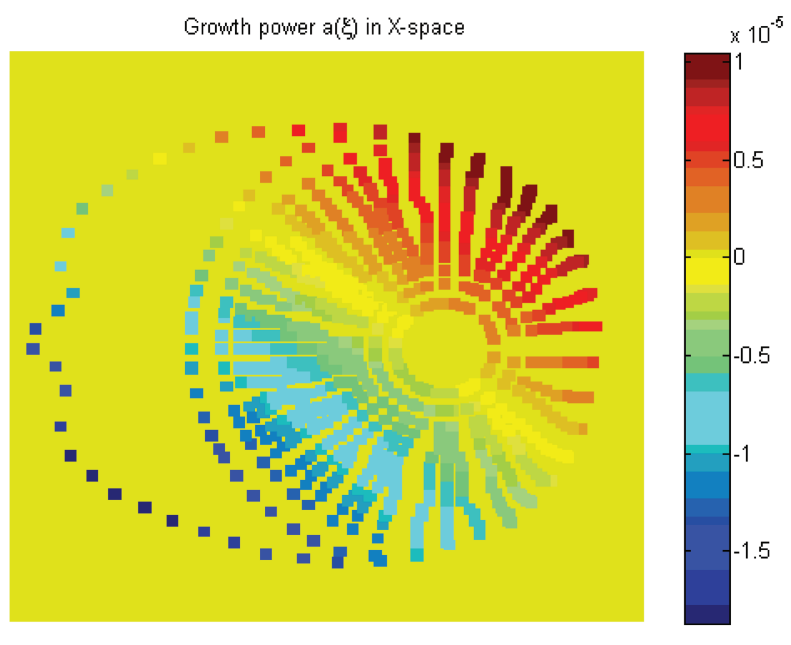

FIG. 18. 
(a): old orgamism with anatomical component in red
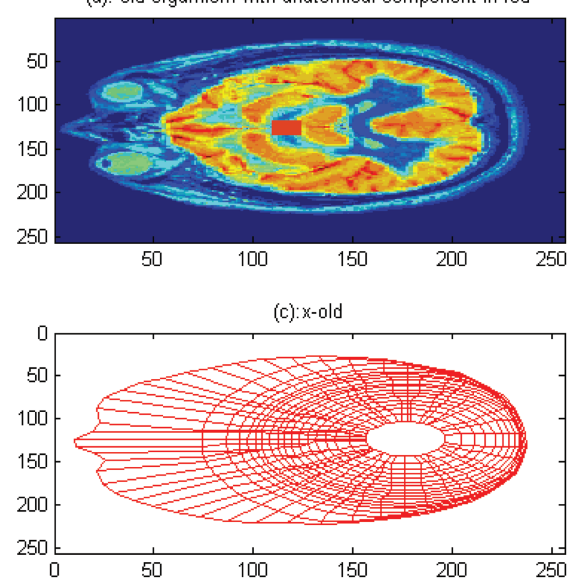

(b) Inferred anatomical component of grown organism in red
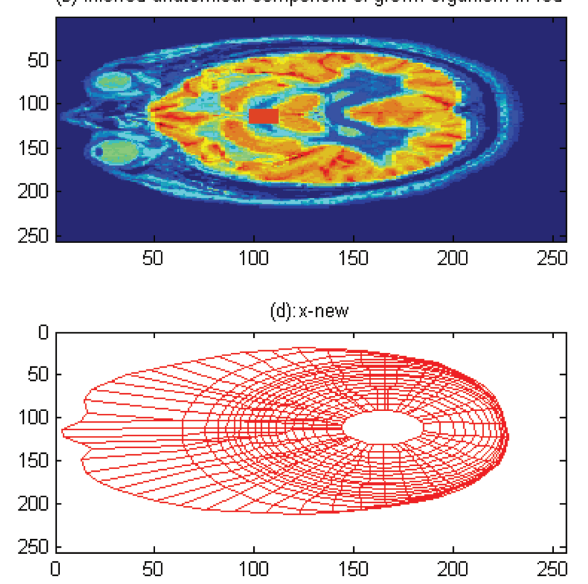

FIG. 19.

\begin{tabular}{|lll|}
\hline 1 & $\rightarrow$ grow & $I 2$ \\
$\downarrow_{\text {anatom }}$ & $\downarrow_{\text {anatom }}$ \\
$\pi 1$ & $\rightarrow$ grow & $\pi 2$ \\
\hline
\end{tabular}

FIG. 20. 


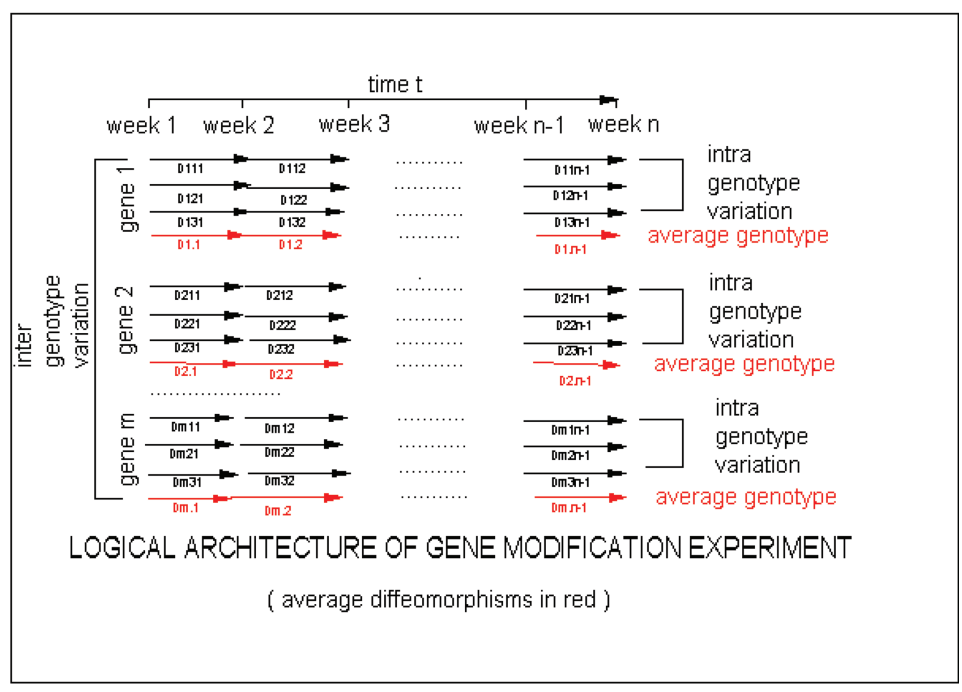

FIG. 21. 


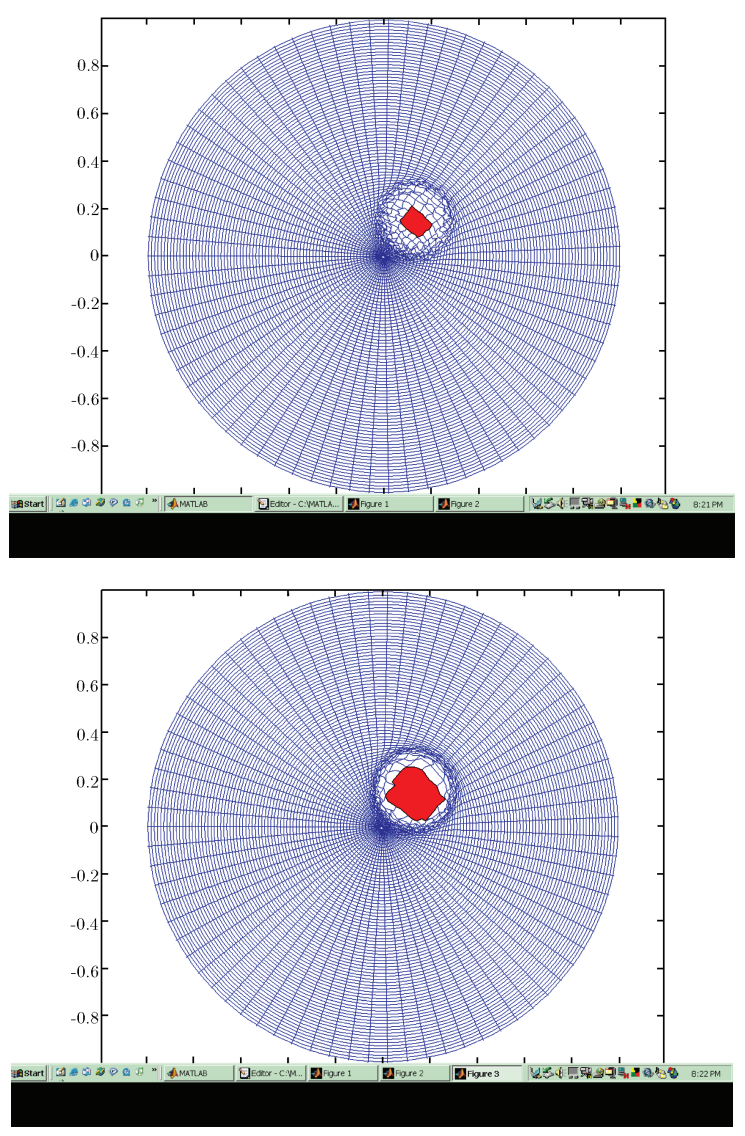

FIG. 22.

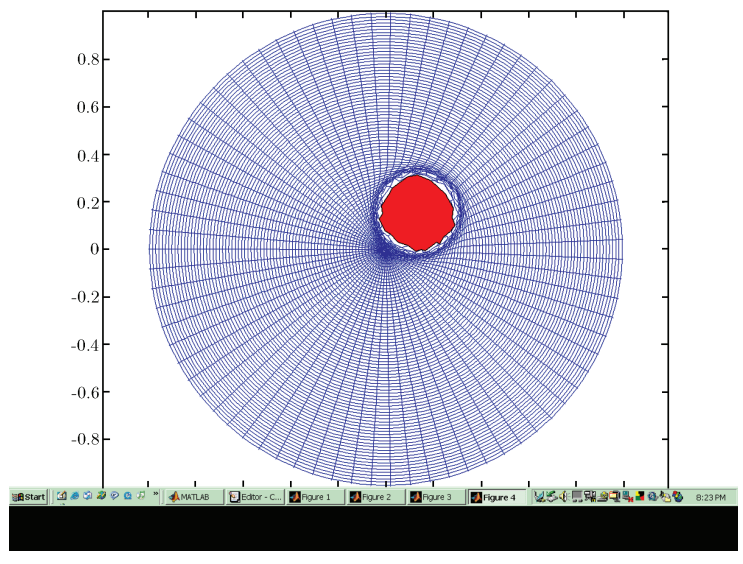

FIG. 23. 
252
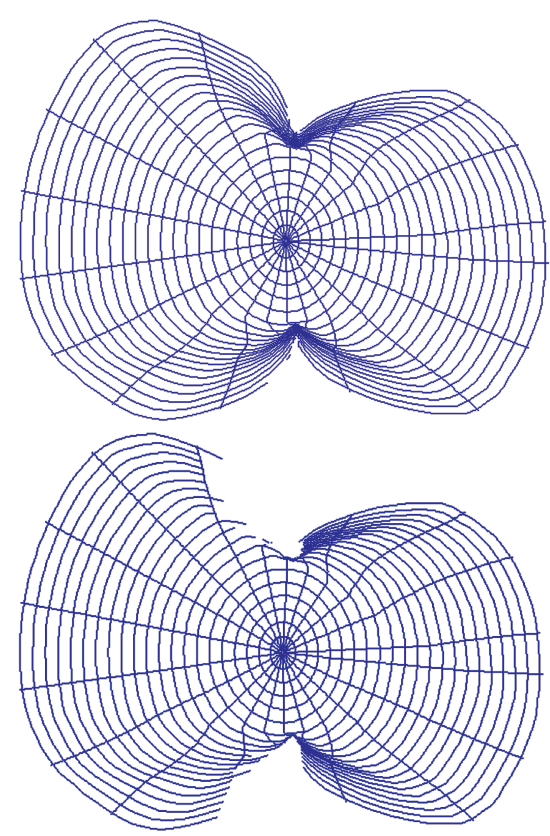

ULF GRENANDER
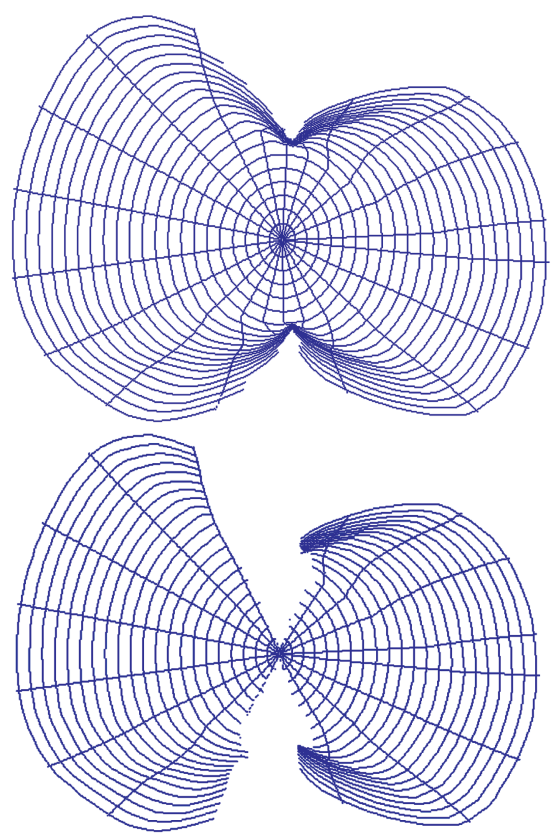

FIG. 24. 
10. Pathogenesis for GRID. So far we have only considered homeomorphic mappings of a template $I_{\text {temp }}$ to the observed image $I^{\mathcal{D}}$; they correspond to normal development of the organism. Now let us turn to pathogenesis, the appearance of pathologies in the development. The two main cases will be the following.

i) CREATION: new material is being created during the development of $\Omega$ so that a part $X_{\text {sing }}$ of $\Omega$ is not covered by the (homeomorphic) mapping; perhaps a tumor is growing.

ii) ANNIHILATION: material of $\Omega$ is being annihilated so that the mapping is not defined on a set $\Xi_{\text {sing }}$ in $\Xi$; perhaps cells are dying.

Let us start with case i) and generate the singular set in $X$ from random seeds as before, but to each seed we now associate a set $S_{\text {seed }} \subset \Xi$, for example a polygon, centered at the seed. The seeds will be chosen from a set $\Xi_{\text {locator }}$ that represents the generation of new material. The union will be the singular set

$$
X_{\text {sing }}=\bigcup_{i} x\left(S_{\text {seed }}^{i}\right)
$$

that will be covered by new material and where $x(\cdot)$ is the current mapping. See the example in Figure 22. where we show the result of a software implementation of the mentioned procedure.

Note that the Darcyan coordinate curves have not been deleted, just pushed away by the new material.

Now case ii). Then we shall choose seeds from a set that we denote as $\Xi_{\text {locator }}$. Then for each seed a region $S_{\text {seed }} \subset \Xi$ will be generated and the Darcyan coordinate curves in

$$
\Xi_{\text {sing }}=\bigcup_{i} S_{\text {seed }}^{i}
$$

will be destroyed together with the corresponding part of $X$. See the example in Figure 23

10.1. GRID in $3 D$. So far we have only considered situations when our observations are two-dimensional. Actually, much of the above carries over to the three-dimensional case with only minor modifications. An exception is the computational realization of these ideas: since 3D data will require much more computer power it will be necessary to devote more attention to the algorithmic aspect than we have done so far.

With this reservation we can leave $3 \mathrm{D}$ to the future. Let us, however, make a remark in passing. Consider an organism in $\mathbf{R}^{3}$, perhaps of elongated form in an embryonic stage, and introduce Darcyan coordinates in the form of a generalized cylinder type $\xi=\left(\xi_{1}, \xi_{2}, \xi_{3}\right)$, where $\xi_{1}$ represents the direction along the longest axis of the organism. Let us consider the singular set $\Xi_{\text {sing }}=\left\{\xi:-l / 2<\xi_{1}<l / 2\right\}$ along the axis of the organism and apply the annihilation mechanism with seeds selected randomly from $\Xi_{\text {sing }}$. This destroys material in a tube along the axis, and we have represented the process of gastrulation that plays such an important role in developmental biology.

11. Inference for pathologies. We shall now turn to a central problem in medical imaging: how to organize inferences drawn from observed images intended to discover 
abnormalities. At this time we can only make some tentative remarks that will prepare the way for a deeper analysis.

To fix ideas let us say that we have access to longitudinal data in the form of pairs of observed images $\left[I_{1}^{\mathcal{D}}\left(t_{1}\right), I_{1}^{\mathcal{D}}\left(t_{2}\right)\right],\left[I_{2}^{\mathcal{D}}\left(t_{1}\right), I_{2}^{\mathcal{D}}\left(t_{2}\right)\right], \ldots,\left[I_{n}^{\mathcal{D}}\left(t_{1}\right), I_{n}^{\mathcal{D}}\left(t_{2}\right)\right]$ for $n$ normal individuals, each observed at durations $t_{1}$ and $t_{2}$. This constitutes the clinical experience of growth for this particular type of organism and for the clinical stratum to which it belongs restricted through limitations on age, gender and other relevant patient specifications.

We are now presented with a new pair $\left[I^{\mathcal{D}}\left(t_{1}\right), I^{\mathcal{D}}\left(t_{2}\right)\right]$ and are asked to infer whether it represents normal growth or growth distributed by some pathology: either creation or annihilation. Hence we are dealing with three hypotheses, one null hypothesis and two alternative hypotheses:

1) $H_{0}$ : normal growth;

2) $H_{\text {creation }}$ : new biological material has been added during the time interval $\left(t_{1}, t_{2}\right)$;

3) $H_{\text {annihilation }}$ : biological material has been deleted during the time interval $\left(t_{1}, t_{2}\right)$.

Since the hypotheses concern the distribution and redistribution of material, it seems natural to use as our observables quantities that describe densities, for example the Jacobians. Here we assume the Jacobians to be calculated from the estimate of $x(\xi, t)$ as discussed in Section 8.3. But we have seen in Section 7.4 that the Jacobians have asymptotically log-normal distributions. We shall therefore associate the three hypotheses with the likelihood functions

$$
\begin{aligned}
& H_{0}: f_{0}\left(I^{\mathcal{D}}\right)=\text { constant } \times \prod_{\xi \in \Xi} \frac{1}{\sigma(\xi) J\left(\xi, t_{2}\right)} \exp \left\{-\sum_{\xi \in \Xi}\left[\log J\left(\xi, t_{2}\right)\right.\right. \\
& \left.-m(\xi)]^{2} /\left(2 \sigma^{2}(\xi)\right)\right\} \text {, } \\
& H_{\text {creation }}: f_{1}\left(I^{\mathcal{D}}, \beta_{\xi}\right)=\text { constant } \times \prod_{\xi \in \Xi} \frac{1}{\sigma(\xi) J\left(\xi, t_{2}\right)} \exp \left\{-\sum_{\xi \in \Xi}\left[\log J\left(\xi, t_{2}\right)\right.\right. \\
& \left.\left.-m(\xi)-\beta_{\xi}\right]^{2} /\left(2 \sigma^{2}(\xi)\right)\right\}, \beta_{\xi}>0, \\
& H_{\text {annihilation }}: f_{2}\left(I^{\mathcal{D}}, \beta_{\xi}\right)=\text { constant } \times \prod_{\xi \in \Xi} \frac{1}{\sigma(x i) J\left(\xi, t_{2}\right)} \exp \left\{-\sum_{\xi \in \Xi}\left[\log J\left(\xi, t_{2}\right)\right.\right. \\
& \left.\left.-m(\xi)+\beta_{\xi}\right]^{2} /\left(2 \sigma^{2}(\xi)\right), \beta_{\xi}>0\right\},
\end{aligned}
$$

with the nuisance parameter $\beta_{\xi}$ forming a scalar field over $\Xi$. This is a direct consequence of the form of the probability density for the log-normal distribution

$$
\frac{1}{\sqrt{2 \pi} \sigma x} \exp \left[-(\log x-m)^{2} /\left(2 \sigma^{2}\right)\right]
$$

We have used the mean values and variances of $\log$ Jacobians under $H_{0}$ and suggest that they be estimated by computing the mappings $x\left(\cdot, t_{1}\right)$ and $x\left(\cdot, t_{2}\right)$ from the samples in the longitudinal data and then estimate the normal parameters $m(\xi)$ by

$$
\frac{1}{n} \sum_{k=1}^{n} \log \operatorname{det}\left[\frac{\partial x^{k}\left(\xi, t_{2}\right)}{\partial x^{k}\left(\xi, t_{1}\right)}\right]
$$

and a similar estimate for the variances $\sigma^{2}(\xi)$. 
REMARK. The above should be taken with some reservation. Indeed, we do not know to what extent the obvious stochastic dependence between various values of $\log J(\xi)$ influences the likelihoods.

We now apply the likelihood ratio test and form critical regions for testing $H_{0}$ against $H_{\text {creation: }}$ :

$$
W=\left\{L_{\text {ratio }}\left(I^{\mathcal{D}}\right)=\frac{\sup _{\beta_{\xi} \geq 0} f_{1}\left(I^{\mathcal{D}}, \beta_{\xi}\right)}{f_{0}\left(I^{\mathcal{D}}\right)}>\text { significance constant }\right\} .
$$

Cancelling terms in the numerator and denominator of the likelihood ratio we get

$$
\log L_{\text {ratio }}\left(I^{\mathcal{D}}\right)=\frac{1}{2 \sigma^{2}} \sum_{\xi \in \Xi} \frac{1}{\sigma(\xi)^{2}} \sup _{\beta_{\xi} \geq 0}\left\{2 \beta_{\xi}\left[\log J\left(\xi, t_{2}\right)-m(\xi)\right]-\beta_{\xi}^{2}\right\} .
$$

But for $\beta \geq 0$,

$$
\begin{gathered}
\max _{\beta}\left[2 a \beta-\beta^{2}\right]=a^{2} \quad \text { for } \quad a>0, \\
\max _{\beta}\left[2 a \beta-\beta^{2}\right]=0 \quad \text { for } \quad a<0 .
\end{gathered}
$$

Hence we have proved the

Proposition. Under given assumptions the likelihood ratio test of $H_{0}$ against $H_{\text {creation }}$ is given by the critical region for the test:

$$
W=\left\{I^{\mathcal{D}}: \sum_{J\left(\xi, t_{2}\right) \geq m(\xi)} \frac{1}{\sigma(\xi)^{2}}\left[\log J\left(\xi, t_{2}\right)-m(\xi)\right]^{2}>\text { constant }\right\} .
$$

A similar expression holds for the testing of $H_{0}$ against the pathological alternative $H_{\text {annihilation }}$.

12. Discussion. The goal in this study has been to incorporate at least the basics of biological growth in the mathematical representation. It must be admitted, however, that this has not been fully achieved, and it is hoped that the GRID formalism will be improved and made more realistic on a detailed level. This can only be done by researchers with real expertise in embryology and developmental biology.

Let us mention some improvements that are clearly indicated.

1) Spelling out the form of the switching function $\alpha(\xi, t)$ in genetic terms, perhaps starting with some quite simple organisms, and in general strengthening and specifying the biological basis for GRID.

2) Applying the inference algorithms to many real growth images. Experiments with animals are useful for this since they can be carried out under controlled laboratory experiments in contrast to data from human patients.

3) Extension to 3D with special emphasis on computational feasibility. This is a matter of software engineering.

4) Extending both the thermodynamic limit and its use for inference by including nonisotropic $k$-functions as described in Section 5.2.2.

5) A more rigorous mathematical analysis of probabilistic limit theorems for GRID, in particular the derivation of the thermodynamic limit as a law of large numbers and of the asymptotic distribution of the Jacobian and other biologically meaningful functionals. 
6) The above discussion of pathogenesis is obviously too little structured; it needs detailed specification and careful data exploration.

7) Deepening the analysis of the construction of Darcyans.

13. Acknowledgments. The author has benefitted from detailed discussions with A. Srivastava who contributed substantially to this study. We are also grateful to D. Khavinson (2005) for informative comments about the potential theoretic question in

Section 4. M. Brewster and B. Gidas helped with the technical layout of the paper. We thank W. Freiberger for accepting the paper for the Quarterly of Applied Mathematics.

\section{REFERENCES}

[1] A. Antoniadis (2003): Optimal Testing in Functional Analysis Models, University of Florida, Department of Statistics, Fifth Annual Winter Workshop.

[2] M. F. Beg, M. I. Miller, A. Trouve, L. Younes (2002): Computing Metrics on Anatomical Shapes in Computational Anatomy. Second Joint Meeting of IEEE EMBS-BMES Society.

[3] J. T. Bonner, ed. (1982): Evolution and Development. Report of the Dahlem Workshop on Evolution and Development, Berlin 1981. Berlin/New York: Springer Verlag.

[4] F. L. Bookstein (1978): The Measurement of Biological Shape and Shape Change. Lecture Notes in Biomathematics, Vol. 24 (Springer Lecture Notes in Computer Science).

[5] F. L. Bookstein (1997): Morphometric Tools for Landmark Data : Geometry and Biology, Cambridge University Press; Reprint edition. MR1469220 (99d:92003)

[6] J. Cao, K.J. Worsley (1999): The detection of local shape changes via the geometry of Hotelling's $\mathrm{T}^{2}$ fields. Annals of Statistics, 27, 925-942. MR1724036 (2001g:60121)

[7] G. E. Christiansen (1994): Deformable shape models for anatomy, Ph.D. Thesis, Washington University.

[8] U. Grenander (1981): Abstract Inference, John Wiley. MR0599175 (82g:62004)

[9] U. Grenander (1993): General Pattern theory, Oxford University Press. MR1270904 (96e:68118)

[10] U. Grenander (2005): Power Point presentation on www.dam.brown.edu/ptg

[11] U. Grenander, M. I. Miller and J. Zhang (2003): FANOVA of genetically modified mouse brains, Div. Appl. Math., Brown University, Rep. No. 3.

[12] U. Grenander, M. I. Miller (1998) Computational anatomy: An emerging discipline, Quarterly of Appl. Math., LVI, 617-694. MR.1668732 (2000e:92011)

[13] A. L. Hodgkin, A. F. Huxley (1952): A quantitative description of membrane current and its application to conduction and excitation in nerve. J. Physiol.

[14] D. Khavinson (2005): oral communication.

[15] D. G. Kendall, D. Barden, T. K. Carne, H. Le (1999): Shape and Shape Theory, Wiley Series in Probability and Statistics. MR1891212 (2003g:60018)

[16] O. Langsrud (2002): 50-50 Multivariate Analysis of Variance for Collinear Responses, The Statistician, 51, 305-317. MR 1920760

[17] R. C. Lewontin (1974): The Genetic Basis of Evolutionary Change, Columbia University Press, New York.

[18] L. Matejic (1997): Group cascades for representing biological variability, Ph.D. Thesis, Brown University.

[19] M. I. Miller (2004): Computational anatomy: Shape, growth, and atrophy comparison via diffeomorphisms, NeuroImage, 23.

[20] M. I. Miller, S. C. Joshi and G. E. Christensen (1999): Large Deformation Fluid Diffeomorphisms For Landmark and Image Matching, Brain Warping, Academic Press.

[21] D. Mumford (2002): Pattern Theory: The Mathematics of Perception. APPTS Report No. 02-10, Div. Appl. Math., Brown University.

[22] N. Rashevsky (1938): Mathematical Biophysics: Physico-Mathematical Foundations of Biology, Univ. of Chicago Press.

[23] A. Srivastava, U. Grenander, S. Saini (2006): Characterization of Biological Growth Using Iterated Diffeomorphisms, Lecture at IEEE International Symposium on Biomedical Imaging. 
[24] d'Arcy Wentworth Thompson (1917, 1942): On Growth and Form. Cambridge University Press. MR0006348(3:291k)

[25] P. M. Thompson, A. W. Toga (2002): A Framework for Computational Anatomy, Comput. Visual. Sci. $5: 13-34$.

[26] R. A. Vitale (1974): Convolutions on a group arising in pattern analysis, Ph.D. Thesis, Brown University.

[27] N. Wiener (1933): The Fourier Integral, Cambridge University Press. 